MAPPING PROPERTIES OF HEAT KERNELS,

MAXIMAL REGULARITY, AND SEMI-LINEAR PARABOLIC EQUATIONS ON NONCOMPACT MANIFOLDS

By

Anna Mazzucato

and

Victor Nistor

IMA Preprint Series \# 2086

(December 2005)

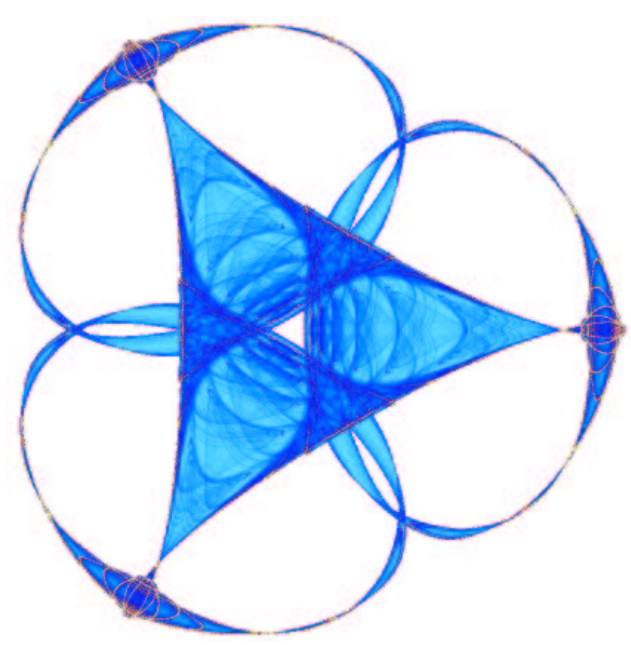

INSTITUTE FOR MATHEMATICS AND ITS APPLICATIONS

UNIVERSITY OF MINNESOTA

400 Lind Hall

207 Church Street S.E.

Minneapolis, Minnesota 55455-0436

Phone: 612/624-6066 Fax: 612/626-7370

URL: http://www.ima.umn.edu 


\title{
MAPPING PROPERTIES OF HEAT KERNELS, MAXIMAL REGULARITY, AND SEMI-LINEAR PARABOLIC EQUATIONS ON NONCOMPACT MANIFOLDS
}

\author{
ANNA MAZZUCATO AND VICTOR NISTOR
}

Version: 1.4; Revised: 03/26/2005 by V.; Run: December 26, 2005

\begin{abstract}
Let $L: \mathcal{C}^{\infty}(M ; E) \rightarrow \mathcal{C}^{\infty}(M ; E)$ be a second order, uniformly elliptic, semi-positive definite differential operator on a complete Riemannian manifold of bounded geometry $M$, acting between sections of a vector bundle with bounded geometry $E$ over $M$. We assume that the coefficients of $L$ are uniformly bounded. Using finite speed of propagation for $L$, we investigate properties of operators of the form $f(\sqrt{L})$. In particular, we establish results on the distribution kernels and mapping properties of $e^{-t L}$ and $(\mu+L)^{s}$. We also show that $L$ generates a holomorphic semigroup that has the usual mapping properties between the $W^{s, p}$-Sobolev spaces on $M$ and $E$. We also prove that $L$ satisfies maximal $L^{p}-L^{q}$ regularity. We apply these results to study parabolic systems of semi-linear equations of the form $\partial_{t} u+L u=F(t, x, u, \nabla u)$.
\end{abstract}

\section{Contents}

Introduction

1. Preliminary results

2. Localization and kernel estimates

3. Mapping properties of heat kernels

4. Maximal $L^{p}-L^{q}-$ regularity

5. Applications to semi-linear parabolic equations -

References

\section{INTRODUCTION}

Let $L$ be a semi-positive definite, uniformly elliptic second order differential operators on a complete Riemannian manifold $M$ of bounded geometry (more precisely, $L$ satisfies Assumptions 1-3 of Section 1). We assume that $L$ acts on sections of a (Hermitian) vector bundle $E \rightarrow M$. We study operators of the form $f(\sqrt{L})$ obtained using functional calculus along with finite propagation speed and localization as in [4].

Localization allows us to approximate $f(\sqrt{L})$ locally with $f\left(\sqrt{L_{\phi}}\right)$, where $L_{\phi}$ are operators on simpler manifolds $\left(\mathbb{R}^{n}\right.$ or the sphere $\left.S^{n}\right)$, which locally identify with $L$ using a local trivialization $\phi$ of $E$ (above a local coordinate chart of $M$ ). This

A. M. was partially supported by NSF grant DMS-0405803. V. N. was partially supported by NSF grant DMS-0200808. 
approach allows us to reduce many estimates on the differential operator $L$ to the analogous estimates on the differential operators $L_{\phi}$, provided that these estimates are independent of $\phi$. This requirement suggests that we should look at estimates on $L$ that are valid for $L$ in a class of operators acting on a class of manifolds with bounded geometry $M$.

Localization and uniform estimates for operators on $\mathbb{R}^{n}$ lead, as in [4], to estimates on the distribution kernels of heat operators $e^{-t L}$, and kernels of the powers $(\mu+L)^{-s}$ of resolvents. In particular, we obtain Gaussian estimates for the heat kernels and exponential decay for the kernels of $(\mu+L)^{-s}$ that are uniform in $L$. Similar results have been established by many authors (see, for example, $[4,5,9,16,25,54])$ in the case that $L$ is the Laplace operator. Exponential decay of distribution kernels of resolvents was proved in this more general setting, using weighted Sobolev spaces $[28,27,41,42]$, also for $L=-\Delta$. (We do not assume that $L=-\Delta$.)

The finite speed of propagation for $L \neq-\Delta$ is more difficult to establish, as there is no natural notion of energy, so we follow [6] here. Considering more general second order operators that Laplaceans is motivated by potential applications to elasticity and incompressible fluid flows.

We use our kernel estimates and the localization principle to prove mapping properties of $e^{-t L}$ between $W^{s, p}$-Sobolev spaces. We also show that $e^{-t L}$ is a holomorphic semigroup and that $L$ satisfies maximal $L^{p}-L^{q}$ regularity for $1<$ $p, q<\infty$, which amounts to well-posedness of the parabolic initial value problem $\partial_{t} u+L u=f, u(0)=g$, for suitable $f$ and $g$ (see Theorem 4.5 for the precise statement).

In Section 5, we apply our results to the study the semi-linear evolution equation (or system)

$$
\partial_{t} u+L u=F(t, x, u, \nabla u), \quad u(0, x)=f(x),
$$

where the non-linearity $F$ satisfies certainb conditions (see Proposition 5.3 and 5.4). Specifically, we consider existence and uniqueness of mild solutions to (1), by recasting the Initial-Value-Problem in integral form. Our approach is based on the Fujita-Kato method [13], owing also to [3, 34, 49].

Equation (1) on $\mathbb{R}^{n}$, on compact manifolds, or on a bounded domain in $\mathbb{R}^{n}$ was extensively studied in the literature, see [12, 47, 49] for instance. Equations of the form (1) model propagation of heat, diffusion-reaction processes appearing in chemistry and biology; it can be used to study Hamilton-Jacobi equations using the viscosity method. By analogy, we expect that Equation (1) on a general, boundedgeometry manifold $M$ could also be useful in describing physical phenomena. For instance, the analysis of (1) could lead to some understanding of relativistic fluids. Direct estensions of the Navier-Stokes systems to 4 dimensions can be seen as first approximation to formulations in hyperbolic form, which respect causality (see $[11,17])$. Since manifolds with cylindrical ends appear naturally when resolving singularities in domains with corners, we also hope that the study of Equation (1) may be useful for understanding similar equations on bounded polyhedral domains and for obtaining numerical methods on these domains.

By $C$ we shall denote a generic positive constant that may change from equation to equation.

We thank Alberto Bressan, Mihalis Dafermos, Sergiu Klainerman, Michael Taylor, and Yuxi Zheng for useful discussions. 


\section{Preliminary Results}

1.1. Assumptions and structural constants. We shall need to study mapping properties of the heat kernel $e^{-t L}$ for a family of elliptic, positive semi-definite, second order differential operators $L$ on a family of smooth manifolds $M$ with metrics $g$ satisfying Assumptions 1, 2, and 3 formulated below. We shall fix $L$ and $M$ in what follows, but we desire estimates that are independent of this choice, for $L$ and $M$ in the given families defined by Assumptions 1, 2, and 3. We assume that $L$ acts between the sections of a smooth vector bundle $\pi: E \rightarrow M$. Before formulating Assumptions 1, 2, and 3, we need to recall some terminology and fix some notation. Non-compact manifolds arise in many practical problems, such as the study of Einstein's field equations $[1,7]$ on asymptotically Euclidean spaces [15], in the AdS-CFT conjecture [14, 26, 29, 32, 53], in the study of singular spaces $[10,18,24,33,36,39,40]$, in the study of the $N$-body problem [51, 52], and in other applications.

Let $M$ be a smooth manifold endowed with a Riemannian metric $g$. For any $x \in M$ and any $v \in T_{x} M$, we shall denote by $\gamma_{v}$ the geodesic such that $\gamma_{v}^{\prime}(0)=v$ (so, in particular, $\gamma_{v}(0)=x$ ). If there exists $r>0$ with the property that $\gamma_{v}(t)$ is defined and minimizing on $\left[0, r^{\prime}\right]$, for any $r^{\prime}<r$, then exists a largest $r$ with this property, called the injectivity radius of $M$ and denoted $r_{\mathrm{inj}}(M)$. We set $r_{\mathrm{inj}}(M)=0$ if there exists no such $r$.

We shall denote by $d(x, y)$ the (geodesic) distance between two points of $M$. Let $B_{t}(x):=\{x \in M, d(y, x)<r\}$ be the open ball of radius $t$ in $M$ centered at $x$. Let $B_{t} \subset \mathbb{R}^{n}$ denote the open ball of radius $t$ centered at the origin. The choice of an orthonormal frame $\xi$ of $T_{x} M$ defines an isometric isomorphism $\mathbb{R}^{n} \rightarrow T_{x} M$. Combining this isomorphism with the geodesic map we obtain the diffeomorphism

$$
\phi_{\xi}: B_{t} \rightarrow B_{t}(x) \text {, for any } 0<t<r_{\text {inj }}(M) .
$$

Let $\left(g_{\alpha \beta}\right)$ be the pull-back via $\phi_{\xi}$ of the metric $g$ to $B_{t}$ and let $\left(g^{\alpha \beta}\right)=\left(g_{\alpha \beta}\right)^{-1}$ be the inverse matrix. Recall that the inverse of $\phi_{\xi}$ is called a normal coordinate system and the matrix $\left(g_{\alpha \beta}\right)$ consists of the components of $g$ in this normal coordinate system.

We are now ready to formulate our assumptions (Assumptions 1, 2, and 3) on the metric $g$ on the smooth manifold $M=M^{n}$ (of dimension $n$ ) and on the differential operator L. The constants appearing in Assumptions 1, 2, and 3 will be called structural constants.

Assumption 1. We assume that there exist positive constants a and $\kappa_{j}, j \geq-1$ such that $r_{i n j}(M)>8 a>0$ and the pull-back metric $g_{\alpha \beta}$ has bounded derivatives and inverse on $B_{7 a}$, that is,

$$
\left|\partial^{\gamma} g_{\alpha \beta}\right| \leq \kappa_{|\gamma|} \quad \text { and } \quad\left|g^{\alpha \beta}\right| \leq \kappa_{-1} \quad \text { on } \quad B_{7 a},
$$

for any choice of the diffeomorphism $\phi_{\xi}: B_{7 a} \rightarrow B_{7 a}(x)$.

The assumption that $M$ has positive injectivity radius implies, in particular, that $M$ is geodesically complete, that is, that all geodesics of $M$ extend indefinitely in time. Let $\nabla:=\nabla_{g}$ be the Levi-Civita connection on $M$ and $R:=R_{g}:=\nabla^{2}$ be its curvature. Assumption 1 also implies that all covariant derivatives $\nabla^{k} R$ are bounded, which together with positive injectivity radius, means, by definition, that 
$M$ has bounded geometry. Let $\psi_{\xi \zeta}$ be the transition function

(3) $\psi_{\xi \zeta}:=\phi_{\zeta}^{-1} \circ \phi_{\xi}: V \rightarrow B_{7 a}$, where $V:=\phi_{\xi}^{-1}\left(B_{7 a}(x) \cap B_{7 a}(y)\right) \subset B_{7 a} \subset \mathbb{R}^{n}$,

between the diffeomorphisms $\phi_{\xi}: B_{7 a} \rightarrow B_{7 a}(x)$ and $\phi_{\zeta}: B_{7 a} \rightarrow B_{7 a}(y)$. Assumption 1 also implies that the transition functions $\psi_{\xi \zeta}$ have bounded derivatives with bounds independent of $\xi$ and $\zeta$. All these bounds depend only on the constants $a$ and $k_{j}$ of Assumption 1. See $[4,27,41,42]$ and there references therein for more details on manifolds of bounded geometry.

Let $E \rightarrow M$ be a hermitian (complex) vector bundle of dimension $\mu$. We shall denote by $\mathcal{C}^{\infty}(M ; E)$ the space of smooth sections of $E$. We shall fix a metric preserving connection $\nabla^{E}: \mathcal{C}^{\infty}(M ; E) \rightarrow \mathcal{C}^{\infty}\left(M ; E \otimes T^{*} M\right)$. Let $x$ be a point in $M$, $\xi$ be an orthonormal basis of $T_{x} M$, and $\eta$ be an orthonormal basis of $E_{x}=\pi^{-1}(x)$. The connection $\nabla^{E}$, together with the normal coordinate system $\phi_{\xi}^{-1}: B_{t}(x) \rightarrow B_{t}$ define a trivialization

$$
\phi=\phi_{\xi \eta}:\left.E\right|_{B_{t}(x)} \rightarrow B_{t} \times \mathbb{C}^{\mu}
$$

via parallel transport along the rays of $B_{t}(x), t<r_{\text {inj }}(M)$. We give the family $\phi_{\xi \eta}$ the smooth structure induced from the smooth structure on the bundle of orthogonal frames of $M$.

Definition 1.1. We shall say that $\left(E, \nabla^{E}\right)$ has bounded geometry if there exist positive constants $\Psi_{j}, j \in \mathbb{Z}_{+}$such that the transition functions $\psi_{j}$ between any two trivializations of the form $\phi_{\xi \eta}$ satisfy $\left|\partial^{\gamma} \psi_{j}\right| \leq \Psi_{|\gamma|}$ for any multi-index $\gamma$.

Let $P: \mathcal{C}_{\mathrm{c}}^{\infty}(M ; E) \rightarrow \mathcal{C}^{\infty}(M ; E)$ be a linear map. Using the trivialization $\phi=\phi_{\xi \eta}$ of Equation (4), we obtain a linear map operator

$$
P_{\phi}: \mathcal{C}_{\mathrm{c}}^{\infty}\left(B_{t}\right) \rightarrow \mathcal{C}^{\infty}\left(B_{t}\right)
$$

$t<r_{\text {inj }}(M)$, by the standard formula $P_{\phi}(f)=[P(f \circ \phi)] \circ \phi^{-1}$, where $f \circ \phi$ is extended to be zero outside $B_{t}(x)$. If $P$ is a differential operator, then $P_{\phi}=\sum_{|\alpha| \leq m} a_{\alpha \phi} \partial^{\alpha}$.

We will consider operators for which $P_{\phi}$ has real-valued coefficients.

Definition 1.2. A differential operator $P: \mathcal{C}_{\mathrm{c}}^{\infty}(M ; E) \rightarrow \mathcal{C}_{\mathrm{c}}^{\infty}(M ; E)$ acting on sections of a vector bundle $E$ with bounded geometry will be said to have uniformly bounded coefficients if there exist positive constants $A_{j}, j \in \mathbb{Z}_{+}$such that, for any trivialization $\phi=\phi_{\xi \eta}$, where $\xi$ is an orthonormal basis of $T_{x} M$ and $\eta$ is an orthonormal basis of $E_{x}$, if we write the operator $P$ in this trivialization as $P_{\phi}=\sum_{|\alpha| \leq m} a_{\alpha \phi} \partial^{\alpha}$, then $\left|\partial^{\gamma} a_{\alpha \phi}\right| \leq A_{|\gamma|}$ on $B_{7 a}$, for any multi-indices $\alpha$ and $\gamma$.

A similar definition leads to the algebras $U \Psi^{\infty}(M ; E)$ of uniformly bounded pseudodifferential operators introduced in [28], provided that one controls also the behaviour of the distribution kernel of $P$ far from the diagonal. See also [41, 42].

Throughout this paper, $L: \mathcal{C}^{\infty}(M ; E) \rightarrow \mathcal{C}^{\infty}(M ; E)$ will denote a second order differential operator.

We recall that a positive semi-definite differential operator $L$ is (uniformly) strongly elliptic if there exists a constant $A_{-1}>0$ such that

$$
-\sum_{|\alpha|=2}\left(a_{\alpha} \eta^{\alpha} v, v\right) \geq A_{-1}|v|^{2}|\eta|^{2},
$$

for any $v \in \mathbb{C}^{\mu}$ and $\eta \in \mathbb{R}^{n}$. 
Assumption 2. We assume that $\left(E, \nabla^{E}\right)$ has bounded geometry and that the second order differential operator $L: \mathcal{C}^{\infty}(M ; E) \rightarrow \mathcal{C}^{\infty}(M ; E)$ has uniformly bounded coefficients (with constants still denoted $\Psi_{j}$ and $A_{j}, j \in \mathbb{Z}_{+}$).

A consequence of Assumption 2 is that the curvature of $\nabla^{E}, R^{E}:=\left(\nabla^{E}\right)^{2} \in$ $\mathcal{C}^{\infty}\left(M ; E \otimes E^{*} \otimes \Lambda^{2} T^{*} M\right)$, is bounded together with all its covariant derivatives with bounds depending only on the structural constants.

Our main example is $E=T^{*} M$ and $L=-\left(a \Delta_{M}+b d d^{*}\right)=-\left(a \Delta_{M}+b \nabla\right.$ div $)$, with $a>0$ and $b \geq 0$ parameters and $\nabla=\nabla_{g}$ the Levi-Civita connection. By $\Delta_{M}$ we denote the Laplace-Beltrami operator on $M$.

We now recall the definition of Soboliev spaces on $E$.

Definition 1.3. Let $k \in \mathbb{Z}_{+}$, then the Sobolev space $W^{k, p}(M ; E)$ is the space of measurable sections $u$ of $E$ such that

$$
\|u\|_{W^{k, p}}^{p}:=\sum_{l=1}^{k} \int_{M}\left|\nabla^{l} u(x)\right|^{p} d x<\infty, \quad 1 \leq p<\infty .
$$

For $p=\infty$ we change this definition in the obvious way, namely we require that,

$$
\|u\|_{W^{k, \infty}}:=\sup \left|\nabla^{l} u(x)\right|<\infty, \quad 0 \leq l \leq k .
$$

If $k<s<k+1$, we define $W^{s, p}(M ; E)$ with norm $\|u\|_{W^{s, p}}$ by complex interpolation,. We write $H^{s}(M ; E)$ for $W^{s, 2}(M ; E)$, and we denote $\cap_{k} W^{k, p}(M ; E)$ by $W^{\infty, p}(M ; E)$. Finally, if $1<p<\infty$, we let $W^{-s, q}(M ; E)=W^{s, p}\left(M ; E^{*}\right)^{*}$, with $p^{-1}+q^{-1}=1$.

When $E=M \times \mathbb{C}$, we simply write $W^{s, p}(M)$. In particular, we let $\mathcal{C}_{b}^{\infty}(M)=$ $\cap W^{k, \infty}(M)$ with the induced family of semi-norms. We shall also write $\|u\|_{L^{p}}=$ $\|u\|_{W^{0, p}}$. We define the Laplacean $\Delta^{E}=\left(\nabla^{E}\right)^{*} \nabla^{E}$. Then $H^{s}(M E)$ identifies with the domain of $\left(1-\Delta^{E}\right)^{s / 2}(M)$, if $s \geq 0$. For $s<0, H^{s}(M ; E)$ identifies with the space of distributions with coefficients in $E^{*}$ that extend by continuity to $H^{|s|}(M ; E),[20]$.

From these definitions, we obtain immediately that a differential operator $P$ of order $m$ defines a bounded map $W^{s, p}(M) \rightarrow W^{s-m, p}(M)$ for $p \in(1, \infty)$ or for $s \geq m$.

Lemma 1.4. Let $P$ be a second order, uniformly strongly elliptic differential operator with uniformly bounded coefficients acting on sections of the vector bundle with bounded geometry $E$. For any $m \in \mathbb{Z}_{+}$we can find constants $a_{m}, a_{m}^{\prime}, b_{m}>0$ that depend only on $m$, on the bounds on the coefficients of $P$, and on the structural constants such that

$$
\left(\left(\Delta^{E}\right)^{m} u, u\right) \leq a_{m}\left(P^{m} u, u\right)+a_{m}^{\prime}(u, u) \leq b_{m}\left(\left(\Delta^{E}\right)^{m} u, u\right)+b_{m}(u, u)
$$

for any $u \in \mathcal{C}_{c}^{\infty}(M ; E)$, the inner product being the $L^{2}$-inner product.

Proof. The first inequality is just the classical Gårding inequality. The key idea is to first prove the inequality in the case of $\mathbb{R}^{n}$, using the Fourier transform. The general case is obtained using a suitable partition of unity to localize to coordinate patches, and the assumption of uniform ellipticity and uniformly bounded coefficients on $P$. See $[6]$ for details of a proof that applies to our framework, following [35]. Since $H^{m}(M ; E)$ is the domain of $\left(\Delta^{E}\right)^{m / 2}$, we have that $\left(\left(\Delta^{E}\right)^{m} u, u\right)+(u, u)$ defines an equivalent norm on $H^{m}(M ; E)$. Since $P^{m}: H^{m}(M ; E) \rightarrow H^{-m}(M ; E)$ 
is continuous, we obtain $\left(L^{m} u, u\right) \leq C\|u\|_{H^{m}}^{2} \leq C\left(\left(\Delta^{E}\right)^{m} u, u\right)+C(u, u)$, which proves the second inequality.

We say that $L$ is positive semi-definite if $(L \phi, \phi) \geq 0$, for any compactly supported section $\phi$ of $E$.

Assumption 3. We shall assume also that $L: \mathcal{C}^{\infty}(M ; E) \rightarrow \mathcal{C}^{\infty}(M ; E)$ is positive semi-definite and uniformly strongly elliptic.

From now on we shall fix a second order, positive semi-definite differential operator L satisfying Assumptions 1, 2, and 3.

We continue to denote by $E$ the pull-back of the vector bundle $E \rightarrow M$ to the product $[0, \epsilon) \times M$, so that the derivative $\partial_{t}$ in the first variable is defined on smooth sections of $E \rightarrow[0, \epsilon) \times M$. The following result is the finite propagation speed for the hyperbolic operator $\partial_{t}^{2}+L$. Recall that the constants of the Assumptions 1, 2, and 3 are called structural constants.

Proposition 1.5. Let $L$ be a second order differential operator satisfying Assumptions 1, 2, and 3, and let $X \subset M$ be a closed set. There exists a constant $c_{M}>0$ that depends only on the structural constants with the following property. Assume that $u \in \mathcal{C}^{\infty}([0, \epsilon) \times M ; E)$ satisfies $\partial_{t}^{2} u(t, y)+L u(t, y)=0$ and that $u(0, y)=\partial_{t} u(0, y)=0$ for any $y \notin X$ and any $t \geq 0$. Then $u(t, x)=0$ for all $x \in M$ such that the distance from $x$ to $X$ is greater than $c_{M} t$.

A proof can be found in [6], Theorem 5.10. The proof there is based on energy estimates for the stress-energy tensor and a (weak) Gårding-type inequality for $L$, which is a direct consequence of uniform strong ellipticity. See [47], Section 2.8, for a simplified proof in the case of of scalar, linear, hyperbolic equations that is in the spirit of $[6]$.

For each fixed $L$ as in the above proposition, there exists a least constant $c_{M}$ satisfying the conclusions of the proposition. We shall denote this constant by $c_{L}$ and call it the propagation speed of $L$.

1.2. First consequences. Let $\delta_{x}$ be the Dirac measure concentrated at $x \in M$. The distribution $\partial^{\alpha} \delta_{x}$ is defined using normal coordinates centered at $x$. Our assumption on the uniform bounded geometry of $M$, Assumptions 1-3, give the following Lemma.

Lemma 1.6. For any multi-index $|\alpha|$ and any $s>|\alpha|+n / 2, \partial^{\alpha} \delta_{x} \in H^{-s}(M)$, and its norm depends only on $s,|\alpha|$, and the structural constants.

As an immediate consequence, we have the following corollary.

Corollary 1.7. The map $(\mu+L)^{s}: H^{m}(M ; E) \rightarrow H^{m-2 s}(M ; E)$ is an isomorphism with $\left\|(\mu+L)^{s}\right\|$ and $\left\|(\mu+L)^{-s}\right\|$ uniformly bounded when $\mu>0$ and $m, s \in \mathbb{R}$ are restricted to compact sets.

Proof. Let us assume first that $m=2 s$. Since $L$ is positive semi-definite, its Friedriechs extension is defined and is a self-adjoint operator on $L^{2}(M ; E)$. Lemma 1.4 shows that the domain of (the Friedriechs extension of) $L$ is $H^{2}(M ; E)$. Therefore, $(\mu+L)^{s}$ is defined by functional calculus for any $s \in \mathbb{R}$ and $\mu>0$. For $s \leq 0$ it is a bounded operator on $L^{2}(M ; E)$ and for $s>0$ it is an unbounded selfadjoint operator. Since the operators $(\mu+L)^{s}\left(\mu^{\prime}+L\right)^{-s}$ are bounded for $\mu, \mu^{\prime}>0$, it follows that $(\mu+L)^{s}$ has the same domain for all $\mu$ and $s \geq 0$ fixed. For 
$\mu=1$ and $m \in \mathbb{Z}_{+}$, this domain is $H^{m}(M ; E)$, by Lemma 1.4. This shows that $(\mu+L)^{m / 2}: H^{m}(M ; E) \rightarrow L^{2}(M ; E)$ is an isomorphism for $m \in \mathbb{Z}_{+}$. The uniform boundedness of the norm of of $(\mu+L)^{m / 2}: H^{m}(M ; E) \rightarrow L^{2}(M ; E)$ for $\mu$ in a compact subset of $(0, \infty)$ follows from the continuity of $(\mu+L)^{s}\left(\mu^{\prime}+L\right)^{-s}$ in the operator norm on $L^{2}(M ; E)$ for $\mu, \mu^{\prime}>0$. This proves the result for $m=2 s \in \mathbb{Z}_{+}$. For arbitrary $m=2 s$, it follows by interpolation.

Finally, the general case follows from the case $m=2 s$ using the isomorphisms $(\mu+L)^{r / 2}: H^{r}(M ; E) \rightarrow L^{2}(M ; E)$ for suitable values of $r \in \mathbb{R}$.

We will partially extend this result to $L^{p}$-type Sobolev spaces in Theorem 3.5. .

Corollary 1.8. The operators $(\mu+L)^{s}: H^{2 s}(M ; E) \rightarrow L^{2}(M ; E), \mu, s \in \mathbb{R}_{+}$, are self-adjoint as unbounded operators on $L^{2}(M ; E)$.

Proof. The result for $\mu>0$ follows from the proof of Corollary 1.7. We have already seen that $L$ is self-adjoint, so $L^{s}$ is self-adjoint if defined using the spectral theorem. Its domain is the same as that of $(1+L)^{s}$ since $(1+L)^{-s} L^{s}$ and $\left(1+L^{s}\right)^{-1}(1+L)^{s}$ are bounded operators. Hence the domain of $L^{s}$ is $H^{2 s}(M ; E)$.

\section{LOCALIZATION AND KERNEL ESTIMATES}

In this section we first review the localization of the functional calculus $f(\sqrt{L})$ of $L$ if $\hat{f}$ is compactly supported. This leads to the usual estimates on the kernels of $e^{-t L}$ and $(\mu+L)^{s}$, the novelty here being that our estimates are uniform in the operator $L$. We do not assume $L=-\Delta$. These estimates lead us to a few preliminary results on the mapping properties of $e^{-t L}$ and $(\mu+L)^{s}$, more precise results being postponed for the next section, Section 3 .

We continue to denote by $M$ a smooth manifold with metric $g$ and by

$$
L: \mathcal{C}^{\infty}(M ; E) \rightarrow \mathcal{C}^{\infty}(M ; E)
$$

a second order differential operator satisfying Assumptions 1, 2, and 3 of the previous subsection for some fixed structural constants $a, \kappa_{j}, \Psi_{j}$, and $A_{j}$. Recall that $L$ has finite speed of propagation $c_{L}$.

We shall denote by $\hat{f}$ the Fourier transform of a tempered distribution. In particular, $\hat{f}(s):=\int_{-\infty}^{\infty} e^{-\imath s t} f(t) d t$ if $f \in L^{1}(\mathbb{R})$. Let $f: \mathbb{R} \rightarrow \mathbb{R}$ be a measurable function. Since $L$ is positive semi-definite and essentially self-adjoint by Corollary 1.8 , we can define $f(\sqrt{L})$ using the spectral theorem $[4,47]$.

We assume that $f$ is even and that $g(x):=\left(\mu^{2}+x^{2}\right)^{-\ell} f(x), \mu>0$, is such that $g, \hat{g} \in L^{1}(\mathbb{R})$ for some $\ell \in \mathbb{Z}_{+}$. Then the properties of the functional calculus of self-adjoint operators give (see [4])

$$
f(\sqrt{L})=\frac{1}{2 \pi}\left(\mu^{2}+L\right)^{\ell} \int_{-\infty}^{\infty} \hat{g}(s) \cos (s \sqrt{L}) d s .
$$

Since $\|\cos (s \sqrt{L})\|=\|\cos (s \sqrt{L})\|_{L^{2} \rightarrow L^{2}} \leq 1$, for $\ell=0$ we obtain immediately that

$$
\|f(\sqrt{L})\| \leq \frac{1}{\pi} \int_{0}^{\infty}|\hat{f}(s)| d s=: \frac{1}{\pi}\|\hat{f}\|_{L^{1}} .
$$

Let us denote by $T(x, y)[23,47]$ the distribution kernel of a continuous, linear map $T: \mathcal{C}_{\mathrm{c}}^{\infty}(M ; E) \rightarrow \mathcal{C}_{\mathrm{c}}^{\infty}(M ; E)^{\prime}$, defined by Schwartz' kernel theorem. We shall typically use this notation when $T(x, y)$ is in fact a function, so the actual values $T(x, y) \in \operatorname{Hom}\left(E_{y}, E_{x}\right)$ are defined for all $x, y \in M$. The support of an operator $T$ 
will be, by definition, the support of its distribution kernel. We then proceed as in [4] to conclude the following.

Proposition 2.1. Assume that the Fourier transform $\hat{f}(s)$ of the even function $f \in \mathcal{S}(\mathbb{R})$ vanishes for $|s| \geq t>0$. Then the support of $f(\sqrt{L})$ is contained in the set $\left\{(x, y) \in M^{2}, d(x, y) \leq c_{L} t\right\}$, where $c_{L}$ is the propagation speed of $L$.

Proof. This is an immediate consequence of Proposition 1.5 and of the fact that $u(t):=\cos (t \sqrt{L}) g, g \in \mathcal{C}_{\mathrm{c}}^{\infty}(M)$, is a smooth function satisfying the equation $u^{\prime \prime}+$ $L u=0$ with initial conditions $u(0)=g$ and $u^{\prime}(0)=0$.

Since the wave operators $\cos (s \sqrt{L})$ are determined locally, we obtain the following result. With abuse of notation, we denote the kernel of the operator $f(\sqrt{L})$ by $f(\sqrt{L})(x, y)$. Recall that $a>0$ is a fixed constant such that $r_{\text {inj }}(M)>8 a$.

Proposition 2.2. Assume that the Fourier transform $\hat{f}(s)$ of the even function $f \in \mathcal{S}(\mathbb{R})$ vanishes for $|s| \geq t$. Then $k_{f}(x, y)$, the restriction of the distribution kernel of $f(\sqrt{L})$ to a small neighborhood of $(x, y) \in M, d(x, y)<b$, depends only on the restriction of $L$ to $B_{2 b}(y)$, for any $0<b<r_{\mathrm{inj}}(M) / 2$ and $t<b / C_{L}$.

Proof. The distribution $k(t, x, y)=\cos (t \sqrt{L})(x, y)$ satisfies, for fixed $y$, the equation $\partial_{t}^{2} k+L k=0$ for $t>0$ with initial conditions $k(0)=\delta_{y}, k^{\prime}(0)=0$. (Here $\delta_{y}$ is Dirac's distribution at the point $y$.) If $L_{1}=L$ in $B_{2 b}(y)$ (the open ball in $M$ with radius $2 b$ centered at $y)$, let $k_{1}(t, x, y)$ be the distribution kernel of $\cos \left(t \sqrt{L_{1}}\right)$, and let $X$ be the complement of the (open) ball $B_{2 b}(y)$. Then $u(t, x):=k_{1}(t, x, y)-k(t, x, y)$ satisfies $\partial_{t}^{2} u(t, x)+L u(t, x)=$ and $u(0, x)=\partial_{t} u(0, x)$ for $x \notin X$. Proposition 1.5 then shows that $u(t, x)=0$ if the distance from $x$ to $X$ is $\geq t C_{L}$. (Even if $u$ is not smooth, we can reduce to this case by replacing $k(t, x, y)$ with $\int_{M} k(t, x, z) \phi(z) d y$, for test function $\phi$ with support close to $y)$. In particular, $u(t, x)=0$ if $d(x, y)<b$ and $t C_{L}<b$. The result now follows from (9), in which we can take $\ell=0$ and $g=f$.

The remaining results in this section are inspired by those in [4]. The shall need the Banach space $\mathcal{F}^{k}$ defined by

$$
\mathcal{F}^{k}:=\left\{f: \mathbb{R} \rightarrow \mathbb{C}, f \text { even and } \hat{f}^{(j)} \in L^{1}(\mathbb{R}) \text { for } j=0,1, \ldots, k\right\} .
$$

The definition of the spaces $\mathcal{F}^{k}$ is similar to that of the spaces $\mathcal{F}_{1}^{k}$ in Equation (1.7) of [4]. The norm on $\mathcal{F}^{k}$ is $\|f\|_{\mathcal{F}^{k}}:=\sum_{j=0}^{k}\left\|f^{(j)}\right\|_{L^{1}\left(\mathbb{R}_{+}\right)}$.

The following theorem gives estimates on the kernel $f(\sqrt{L})$ and is completely analogous to Theorem 1.4 of [4]. The norm in $\left\|\partial_{x}^{\alpha} \partial_{y}^{\beta} f(\sqrt{L})(x, y)\right\|$ below refers to the (Hilbert space) norm on $\mu \times \mu$ matrices. The derivatives $\partial_{x}^{\alpha}$ and $\partial_{y}^{\beta}$ are with respect to normal coordinates systems of the form $\phi_{\xi}$ defined in Equation (2). Note that we also need the trivializations $\phi_{\xi \eta}$ of Equation (4) in order to identify $\partial_{x}^{\alpha} \partial_{y}^{\beta} f(\sqrt{L})(x, y)$ with an $\mu \times \mu$ matrix (recall that $\mu$ is the dimension of the vector bundle $E$ on whose sections $L$ acts).

Theorem 2.3. Let $c_{L}$ be the propagation speed of $L$, let $f \in \mathcal{F}^{N}, N$ even, and $r \leq s+N / 2$. Then:

(i) $f(\sqrt{L})$ maps $H^{s}(M ; E)$ to $H^{r}(M ; E)$ with $\|f(\sqrt{L})\|_{H^{s} \rightarrow H^{r}} \leq C\|f\|_{\mathcal{F}^{N}}$. 
(ii) Let $b=0$ if $d(x, y) \leq a$ and $b c_{L}=d(x, y)-a$ otherwise. Then,

$$
\left|\partial_{x}^{\alpha} \partial_{y}^{\beta} f(\sqrt{L})(x, y)\right| \leq C \sum_{j=0}^{N} \int_{b}^{\infty}\left|\hat{f}^{(j)}(t)\right| d t, \quad|\alpha|+|\beta| \leq N-n-4 .
$$

Proof. The proof is very similar to that of Theorem 1.4 in [4], using Equation (9), that $M$ has bounded geometry, that the $L^{2}$-norm $\|\cos (t \sqrt{L})\|=1$, and that $L$ has propagation speed $C_{L}$. We sketch the main steps for the benefit of the reader.

Again, let $\|T\|=\|T\|_{L^{2} \rightarrow L^{2}}$ be the norm of an operator acting on $L^{2}(M ; E)$. Equation (10) and the relation $\left|\widehat{x^{2 j} f}(t)\right|=\left|\hat{f}^{(2 j)}(t)\right|$ give right away the following simplified version of Corollary 1.2 of [4]

$$
\left\|L^{k} f(\sqrt{L}) u\right\|=\left\|f(\sqrt{L}) L^{k} u\right\|_{L^{2} \rightarrow L^{2}} \leq \frac{\|u\|_{L^{2}}}{\pi} \int_{0}^{\infty}\left|\hat{f}^{(2 k)}(t)\right| d t .
$$

Corollary 1.7 then implies the norm estimates

$$
\begin{aligned}
\|f(\sqrt{L})\|_{H^{s} \rightarrow H^{r}} \leq & C\left\|(1+L)^{r / 2} f(\sqrt{L})(1+L)^{-s / 2}\right\|=\left\|(1+L)^{(r-s) / 2} f(\sqrt{L})\right\| \\
& \leq\left\|(1+L)^{N / 2} f(\sqrt{L})\right\| \leq C \sum_{j=0}^{N / 2} \int_{0}^{\infty}\left|\hat{f}^{(2 j)}(t)\right| d t \leq C\|f\|_{\mathcal{F}^{N} .}
\end{aligned}
$$

This proves the first part of our result.

Next we use that $\partial_{x}^{\alpha} \partial_{y}^{\beta} f(\sqrt{L})(x, y)=(-1)^{|\beta|}\left\langle\partial^{\alpha} \delta_{x}, f(\sqrt{L}) \partial^{\beta} \delta_{y}\right\rangle$. Therefore, for $s>n / 2$, the same calculation yields

$$
\begin{array}{r}
\left|\partial_{x}^{\alpha} \partial_{y}^{\beta} f(\sqrt{L})(x, y)\right| \leq C\|f(\sqrt{L})\|_{H^{-s-|\beta|} \rightarrow H^{s+|\alpha|}}\left\|\partial^{\alpha} \delta_{x}\right\|_{H^{-(s+|\alpha|)}}\left\|\partial^{\beta} \delta_{y}\right\|_{H^{-(s+|\beta|)}} \\
\leq C\left\|(1+L)^{k} f(\sqrt{L})(1+L)^{-l}\right\|_{L^{2} \rightarrow L^{2}} \leq C \sum_{j=0}^{2 k+2 l} \int_{0}^{\infty}\left|\hat{f}^{(j)}(t)\right| d t,
\end{array}
$$

provided that $2 k \geq s+|\alpha|$ and $2 l \geq s+|\beta|$. This proves the result if $d(x, y) \leq a$.

Let $\chi \in \mathcal{C}^{\infty}(\mathbb{R})$ be a fixed smooth function such that $0 \leq \chi \leq 1, \chi(t)=1$ for $t \leq 0, \chi(t)=0$ for $t \geq a / c_{M}$. If $d(x, y)>a$, let $b c_{L}=d(x, y)-a$ be as in the statement and write $f=f_{1}+f_{2}$, where $f_{1}$ and $f_{2}$ are even functions such that $f_{1}(t)=f(t) \chi(t-b)$ for $t \geq 0$ (this completely determines $f_{1}$, since $f_{1}$ is even). Then $f(\sqrt{L})=f_{1}(\sqrt{L})+f_{2}(\sqrt{L})$ and the support of $f_{1}(\sqrt{L})$ is contained in the set of points $\left(x^{\prime}, y^{\prime}\right) \in M^{2}$ at distance at most $b C_{M, L}+a$. Consequently $f_{1}(\sqrt{L})(x, y)=0$, and hence

$$
\begin{aligned}
\left\|\partial_{x}^{\alpha} \partial_{y}^{\beta} f(\sqrt{L})(x, y)\right\|=\left\|\partial_{x}^{\alpha} \partial_{y}^{\beta} f_{2}(\sqrt{L})(x, y)\right\| \leq C \sum_{j=0}^{N} \int_{0}^{\infty}\left|\hat{f}_{2}^{(j)}(t)\right| d t & \\
& \leq C \sum_{j=0}^{N} \int_{b}^{\infty}\left|\hat{f}^{(j)}(t)\right| d t .
\end{aligned}
$$

The proof is now complete.

Since $\mathcal{S}(\mathbb{R}) \subset \mathcal{F}^{N}$ for all $N$, an immediate consequence of Theorem 2.3 is the following corollary.

Corollary 2.4. If $f \in \mathcal{S}(\mathbb{R})$, then $f(\sqrt{L})$ is a regularizing operator; that is, it maps $H^{-s}(M ; E) \rightarrow H^{s}(M ; E)$ for any $s>0$. 
We now turn to localization results for operators of the form $f(\sqrt{L})$, which include estimates on the heat kernels. Let $0<8 a<r_{i n j}(M)$, as before. Also, let $\chi \in \mathcal{C}_{\mathrm{c}}^{\infty}(\mathbb{R})$ be an even function with support in $\left[-2 a / c_{M}, 2 a / c_{M}\right]$ and such that $\chi=1$ on $\left[-a / c_{M}, a / c_{M}\right]$. We shall decompose any even function $f \in \mathcal{S}^{\prime}(\mathbb{R})$ as

$$
f=f_{1}+f_{2}, \quad \hat{f}_{1}=\chi \hat{f}
$$

and call this the finite propagation speed decomposition of $f$. Clearly, $f_{1}, f_{2} \in \mathcal{S}(\mathbb{R})$ if $f \in \mathcal{S}(\mathbb{R})$. In all the examples considered below, $f$ will be a classical symbol on $\mathbb{R}$, and hence $f_{2} \in \mathcal{S}(\mathbb{R})$. From now on, we assume that $f \in S_{c l}^{\infty}(\mathbb{R})$ (i.e., that $f$ is a classical symbol).

The choice of the function $\chi$ will influence the constants appearing in the statements below. However, since $\chi$ will remain fixed, we shall not indicate the dependence of the various constants on $\chi$. The properties of $f_{1}(\sqrt{L})$ are easily established using the previous results (for example, finite propagation speed).

Consider first a fixed, but arbitrary trivialization $\phi=\phi_{\xi \eta}:\left.E\right|_{B_{7 a}(x)} \rightarrow B_{t} \times \mathbb{C}^{\mu}$ as in Equation (4) and let $L_{\phi}$ be the differential operator induced by $L$ on $B_{7 a} \times \mathbb{C}^{\mu} \subset$ $\mathbb{R}^{n} \times \mathbb{C}^{\mu}$. We chose an extension $\mathcal{L}_{\phi}$ of $L_{\phi}$ to a positive semi-definite, uniformly strongly elliptic differential operator on $\mathbb{R}^{n}$ with smooth coefficients that satisfy the same conditions as the coefficients of $L$ with slightly larger constants. This can be realized by first replacing $L_{\phi}$ with $\chi_{0} L_{\phi} \chi_{0}+\nu \Delta$, where $\chi_{0} \mathcal{C}_{\mathrm{c}}^{\infty}\left(\mathbb{R}^{n}\right)$ has support in $B_{7 a}$ and is equal to 1 on $B_{6 a}$. That is, eventually by changing the structural constants, we can achieve that both $(M, E, L)$ and $\left(\mathbb{R}^{n}, \mathbb{C}^{\mu}, \mathcal{L}_{\phi}\right)$ satisfy Assumptions 1,2 , and 3 . Occasionally (in Theorem 2.5, for instance) we shall consider $\mathcal{L}_{\phi}$ to be operators on a sphere instead of $\mathbb{R}^{n}$, which can be defined using the same procedure.

The following result was proved for $L=-\Delta$ in [4] and [42].

Theorem 2.5. Let $\mathcal{L}_{\phi}$ be the fixed extension of $L_{\phi}$. Also, let $f=f_{1}+f_{2}$ be the finite propagation speed decomposition of some $f \in S_{c l}^{s}(\mathbb{R}), s \in \mathbb{R}$, as in Equation (12). Then, for all $x, y \in M$ such that $d(x, y) \leq 3 a$,

$$
f_{1}(\sqrt{L})(x, y)=f_{1}\left(\sqrt{\mathcal{L}_{\phi}}\right)(x, y) .
$$

The family $f_{1}\left(\sqrt{\mathcal{L}_{\phi}}\right)$ is a bounded family of pseudodifferential operators of order $m$ depending smoothly on $\phi$. Moreover, $f_{1}(\sqrt{L})(x, y)$ depends only on $L_{\phi}$, and $f_{1}(\sqrt{L})(x, y)=0$ if $d(x, y) \geq 2 a$.

Proof. The theorem, except establishing that the family $f_{1}\left(\sqrt{\mathcal{L}_{\phi}}\right)$ is a bounded family of pseudodifferential operators of order $m$, follows from Proposition 2.2 with $t=2 a / C_{M}$ and $b=3 a$.

Since $f_{2} \in \mathcal{S}(\mathbb{R})$, Corollary 2.4 shows that $f_{2}(\sqrt{L})$ has a smooth distributional kernel. We can hence assume that $f=f_{1}$. Since $f$ is an even classical symbol, it can be written as the asymptotic sum of symbols of the form $\left(1+x^{2}\right)^{(s-j) / 2}$. Since $\left(1+\mathcal{L}_{\phi}\right)^{(s-j) / 2}$ is a bounded family of pseudodifferential operators [45], we obtain that $f\left(\sqrt{\mathcal{L}_{\phi}}\right)$ is pseudodifferential operator. Corollary 2.4 allows us to reduce our calculation to a symbolic one. Then, using that the functional calculus depends smoothly on $\mathcal{L}_{\phi}$, we obtain that the family $f\left(\sqrt{\mathcal{L}_{\phi}}\right)$ is also bounded and that it depends smoothly on $\phi$.

The equality $f_{1}(\sqrt{L})(x, y)=f_{1}\left(\sqrt{\mathcal{L}_{\phi}}\right)(x, y)$ should be understood in the following sense. The trivialization $\phi_{\xi \eta}$ of Equation (4) used to define $L_{\phi}$ will map the restriction of the distribution kernel of $f_{1}(\sqrt{L})$ to the ball $d(x, y) \leq 3 a$ ( $x$ is fixed) to 
the corresponding restriction of the distribution kernel of $f_{1}\left(\sqrt{L_{\phi}}\right)$. In particular, one can say $f_{1}(\sqrt{L})_{\phi}=f_{1}\left(\sqrt{\mathcal{L}_{\phi}}\right)$ on a ball of radius $3 a$ around $x$, so in a certain sense localization commutes with functional calculus by $f_{1}$ for suitable $f_{1}$.

We now extend the previous result to complex functions $f: \mathbb{C} \rightarrow \mathbb{C}$. This extension is necessary in order to show that $L$ generates a holomorphic semigroup $e^{z L}$ in the next section.

Let us denote by $\Xi_{\theta} \subset \mathbb{C}$ the sector

$$
\Xi_{\theta}:=\{0<|z| \leq 1, \Re(z) \geq \theta|z|\}, \quad \theta>0 .
$$

Theorem 2.6. Let $t \in \Xi_{\theta}, f(x)=e^{-t x^{2}}$, and $f=f_{1}+f_{2}$ be the finite propagation speed decomposition of $f$, Equation (12). Then for any $\epsilon \in(0,1 / 4]$ and $N \in \mathbb{Z}_{+}$, we have that the distribution kernel $f_{2}(\sqrt{L})(x, y)$ of $f_{2}(\sqrt{L})$ satisfies

$$
\left|\partial_{x}^{\alpha} \partial_{y}^{\beta} f_{2}(\sqrt{L})(x, y)\right| \leq C|t|^{N} e^{-R}, \quad R=\frac{1-\epsilon}{4 c_{M}^{2} \Re(t)} \max \left\{(d(x, y)-a)^{2}, a^{2}\right\},
$$

where $C$ may depend on $N, \alpha, \beta, \epsilon, \theta$, and the structural constants, but not on $t \in \Xi_{\theta}$ or on $x, y \in M$.

Proof. The result is a consquence of Theorem 2.3 applied to the function $f_{2}$. We use also that that $\hat{f}(x)=(4 \pi t)^{-1 / 2} e^{x^{2} /(4 t)}$ and that

$$
\int_{a / c_{M}}^{\infty} t^{-N}|x|^{2 N} e^{-\epsilon|x|^{2} /(8 t)} d x \leq C
$$

an estimate valid for all $t \in \Xi_{\theta}$ and $|x| \geq a$, for some constant $C$ that may depend on $N, a$, and $\epsilon$, but not on $t$.

The next corollary is often enough in applications. We continue to denote by $\Xi_{\theta}$ the sector defined by Equation (13).

Corollary 2.7. Let $t \in \Xi_{\theta}, f(x)=e^{-t x^{2}}$, and $f=f_{1}+f_{2}$ be the finite propagation speed decomposition of $f$, Equation (12). Then, for any $\tau \geq 0$ and $N \in \mathbb{Z}_{+}$we have that the distribution kernel $f_{2}(\sqrt{L})(x, y)$ of $f_{2}(\sqrt{L})(x, y)$ satisfies

$$
\left|\partial_{x}^{\alpha} \partial_{y}^{\beta} f_{2}(\sqrt{L})(x, y)\right| \leq C t^{N} e^{-\tau d(x, y)}
$$

for a constant that may depend on $N, \alpha, \beta$, or $\tau$, but not on $t \in \Xi_{\theta}$.

Proof. Let $R:=\left(4 c_{M}^{2} \Re(t)\right)^{-1}(1-\epsilon) \max \left\{(d-a)^{2}, a^{2}\right\}$, by analogy with Theorem 2.6. The result follows right away from Theorem 2.6 and the inequality $e^{-R} \leq$ $C e^{-\tau d}$, valid for all $d \in \mathbb{R}$, for a constant $C$ that may depend on $\tau$ and $\epsilon>0$ but not on $d$ or on $t \in \Xi_{\theta}$.

We now use a similar analysis to obtain estimates on the distribution kernel $(\mu+L)^{s}(x, y)$ of $(\mu+L)^{s}, s \in \mathbb{R}$, where $(\mu+L)^{s}$ is defined using the spectral theorem.

Theorem 2.8. Let $\mu, \epsilon>0$ and $s \in \mathbb{R}$. Also, let $f(x)=\left(\mu^{2}+x^{2}\right)^{s}$, and $f=f_{1}+f_{2}$ be the finite propagation speed decomposition of $f$, Equation (12).

(i) $f_{1}(\sqrt{L})(x, y)=0$ if $\{d(x, y)>2 a\}$ and $f_{1}(\sqrt{L})_{\phi}$ is a bounded family of pseudodifferential operators depending smoothly on the trivialization $\phi=\phi_{\xi \eta}$.

(ii) The distribution kernel $f_{2}(\sqrt{L})(x, y)$ of $f_{2}(\sqrt{L})$ satisfies

$$
\left|\partial_{x}^{\alpha} \partial_{y}^{\beta} f_{2}(\sqrt{L})(x, y)\right| \leq C e^{-R}, \quad R=(\mu-\epsilon) \max \{d(x, y), a\} / c_{M},
$$


for a constant that may depend on $\alpha, \beta, \epsilon, \mu$, and the structural constants, but not on $x, y \in M$.

Proof. The first part follows from Theorem 2.5. The proof of the second part is completely analogous to that of Theorem 2.6, using also that $|\hat{f}(t)| \leq C e^{-(\mu-\epsilon)|t|}$, since $f$ extends to a holomorphic function on $\{|\Im(z)|<\mu\}$. (See [23], for example, for a proof of this elementary result).

Remark 2.9. If $f(x)=\left(z^{2}+x^{2}\right)^{-1}$ (so $s=-1$ ), then we allow $z \in \mathbb{C}, \Re(z)>0$. In this case, we can explicitly compute the Fourier transform as $\hat{f}(t)=\frac{\pi}{\mu} e^{-z|t|}$, which leads to the more precise estimate

$$
\left|\partial_{x}^{\alpha} \partial_{y}^{\beta} f_{2}(\sqrt{L})(x, y)\right| \leq C|z|^{N-1} e^{-R}, \quad R=(\Re(z)-\epsilon) \max \{d(x, y), a\} / c_{M},
$$

with $C$ independent of $z$, this time. In fact, $C$ depends only on $\alpha, \beta, \epsilon$, and the structural constants, but not on $x, y \in M$ or $z$.

Remark 2.10. By taking the family of manifold $\{M\}$ to consist of a single manifold $M$, we can replace $c_{M}$ with $c_{L}$ in Theorems 2.6 and 2.8 .

Estimates on the heat kernel and the resolvent are now easy to obtain. Similar estimates have been obtained in $[4,27,41,54,5]$ and by others for $L=-\Delta$.

Corollary 2.11. Let $t \in \Xi_{\theta}, \Re(z)>0$, and $N=|\alpha|+|\beta|+n+4$. Then there exists a constant $C>0$ such that for any $x, y \in M, d(x, y) \geq 2 a$, we have

$$
\begin{gathered}
\left|\partial_{x}^{\alpha} \partial_{y}^{\beta} e^{-t L}(x, y)\right| \leq C e^{-R}, \quad R=\frac{1-\epsilon}{4 c_{M}^{2} \Re(t)}(d(x, y)-a)^{2} \\
\left|\partial_{x}^{\alpha} \partial_{y}^{\beta}\left(\mu^{2}-L\right)^{-1}(x, y)\right| \leq C|z|^{N-1} e^{-R_{0}}, \quad R_{0}=(\Re(z)-\epsilon) d(x, y) / c_{M} .
\end{gathered}
$$

Proof. The corollary follows from Theorems 2.6 and 2.8 using the fact that the kernel of $f_{1}(\sqrt{L})$ is supported in the set $\{d(x, y) \leq 2 a\}$, by Proposition 2.1.

Let us recall that there exists a constant $K>0$ such that

$$
\operatorname{vol}\left(B_{r}(x)\right) e^{-K r} \leq C
$$

for a constant $C>0$ that is independent of $x \in M$ and $r>0$. (In fact, it is enough that the Ricci curvature be bounded from below, see [4], page 39.) This observation leads to the following mapping properties of kernels with exponential decay.

Proposition 2.12. Assume that $\tau>K$, where $K$ is the constant of Equation (14) and that the measurable function $k: M^{2} \rightarrow \mathbb{C}$ satisfies $\left|\partial_{x}^{\alpha} k(x, y)\right| \leq C_{0} e^{-\tau d(x, y)}$ for all $|\alpha| \leq m, m \in \mathbb{Z}_{+}$. Then $k$ defines a bounded map $T_{k}: L^{p}(M) \rightarrow W^{m, p}(M)$, for any $1 \leq p \leq \infty$ by the formula $T_{k} \phi(x)=\int_{M} k(x, y) \phi(y) d y$. A similar result holds in the case of sections of a bundle $E \rightarrow M$ with bounded geometry, where $k: M^{2} \rightarrow \mathbb{C}^{\mu} \times \mathbb{C}^{\mu}$.

Proof. It is enough to show that $\nabla^{m} T$ defines a continuous map $L^{p}(M) \rightarrow L^{p}(M)$. To this end, we observe that

$$
\begin{aligned}
\int_{M}\left|\partial^{\alpha} k(x, y)\right| d y & \leq \int_{M} e^{-\tau d(x, y)} d y=\sum_{j} \int_{B_{j+1}(x) \backslash B_{j}(x)} e^{-\tau d(x, y)} d y \\
& \leq \sum_{j} e^{K(j+1)} e^{-\tau j}<\infty
\end{aligned}
$$


and, similarly, $\int_{M} e^{-\tau d(x, y)} d x<\infty$. The result then follows from the classical Riesz lemma (see for instance [45], Chapter XIII).

We shall need the following consequence.

Corollary 2.13. Assume that $\tau>K$, where $K$ is the constant of Equation (14) and that the measurable function $k: M^{2} \rightarrow \mathbb{C}$ satisfies $\left|\partial_{x}^{\alpha} \partial_{y}^{\beta} k(x, y)\right| \leq C_{\alpha \beta} e^{-\tau d(x, y)}$ for all $\alpha$ and $\beta$. Then $k$ defines a bounded map $T_{k}: W^{r, p}(M) \rightarrow W^{r-s, p}(M)$, for any $1<p<\infty$ and any $s, r \in \mathbb{R}$. A similar result holds in the case of sections of a bundle $E \rightarrow M$ with bounded geometry.

Proof. We know that $T_{k}: L^{p}(M) \rightarrow W^{m, p}(M)$ is bounded for any $m \in \mathbb{Z}_{+}$by Proposition 2.12. Let $q$ be the conjugate exponent to $p$ (i.e., $1 / p+1 / q=1)$. By duality $T_{k}: W^{-m, q}(M) \rightarrow L^{q}(M)$ is also bounded for any $m \in \mathbb{Z}_{+}$and any $1<q<\infty$. By interpolation, $T_{k}: W^{-m / 2, p}(M) \rightarrow W^{m / 2, p}(M)$ is then bounded for all $m \in \mathbb{Z}_{+}$and all $1<p<\infty$.

The proof of Proposition 2.12 shows that the norm of the operator $T_{k}$ can be bounded by $C C_{0}$, where $C_{0}$ is the constant appearing in Proposition 2.12 and $C$ is a constant that depends only on $M$. Let $\mathcal{L}\left(L^{p}(M ; E)\right)$ be the space of bounded operators on $L^{p}(M ; E)$. Let us define also, similarly to the space $\mathcal{F}^{k}$ introduced before,

$$
\mathcal{F}_{\tau}^{k}:=\left\{f: \mathbb{R} \rightarrow \mathbb{C}, f \text { even and } e^{\tau|x|} \hat{f}^{(j)}(x) \in L^{1}(\mathbb{R}) \text { for } j=0,1, \ldots, k\right\} .
$$

with norm $\|f\|_{\mathcal{F}_{\tau}^{k}}:=\sum_{j=0}^{k} \int_{0}^{\infty} \hat{e}^{\tau x}\left|f^{(j)}(x)\right| d x$.

Proposition 2.14. Let $N \geq n+4$ and $f \in \mathcal{F}_{\tau}^{N}$. Then $|f(\sqrt{L})(x, y)| \leq C e^{-\tau d(x, y)}$ and hence $f(\sqrt{L}) \in \mathcal{L}\left(L^{p}(M ; E)\right)$ for $\tau>K$, where $K$ is the constant of Equation (14). Moreover, the map $\mathcal{F}_{\tau}^{N} \ni f \rightarrow f(\sqrt{L}) \in \mathcal{L}\left(L^{p}(M ; E)\right)$ is continuous for $\tau>K$.

Proof. We have that $\sum_{j=0}^{N} \int_{x}^{\infty}\left|\hat{f}^{(j)}(t)\right| d t \leq e^{-\tau x}\|f\|_{\mathcal{F}_{\tau}^{N}}$ for $N>0$. The result then follows from Theorem 2.3 (ii) and Proposition 2.12.

\section{MAPPing PROPERTIES OF HEAT KERNELS}

We now establish the needed estimates for the norms of the heat kernels $e^{-t L}$ : $W^{s, p}(M ; E) \rightarrow W^{r, q}(M ; E), s \leq r$ and $1<p \leq q<\infty$. The end values $p, q=1, \infty$ are allowed as long as $s=0$ and $r \in \mathbb{Z}_{+}$, and sometimes for other values as well. These estimates are analogous to the estimates listed on page 274 of [49].

We first need to review an alternative description of the spaces $W^{k, p}(M)$ using partitions of unity as in [4] or [41, Lemma 1.3]. See also [4, 42, 44]. Recall that $0<8 a<r_{i n j}(M)$, by Assumption 1 .

Lemma 3.1. There exist an integer $\kappa$, a sequence of points $\left\{x_{j}\right\} \subset M$, and a partition of unity $\eta_{j} \in \mathcal{C}_{c}^{\infty}(M)$ with the following properties:

(i) $\operatorname{supp}\left(\eta_{j}\right) \subset B_{a}\left(x_{j}\right)$;

(ii) $\left\|\nabla^{k} \eta_{j}\right\|_{L^{\infty}(M)} \leq C_{k}$, with $C_{k}$ independent of $j$; and

(iii) A point $x \in M$ can belong to at most $\kappa$ of the balls $B_{3 a}\left(x_{j}\right)$.

Proof. This is an immediate consequence of Lemmas 1.2 and 1.3 of [41]. 
Let $\phi_{j}=\phi_{\xi_{j}}: B_{7 a}\left(x_{j}\right) \rightarrow B_{7 a}(0)$ be the normal coordinate system around $x_{j}$ corresponding to a choice of an orthonormal frame $\xi_{j}$ at $x_{j} \in M$, as in Equation (2). The following result was proved in [50].

Lemma 3.2. Let the partition of unity $\left\{\eta_{j}\right\}$ and the normal coordinate systems $\left\{\phi_{j}\right\}$ be as described above. Let $B=B_{2 \epsilon}(0)=\phi_{j}\left(B_{2 \epsilon}\left(x_{j}\right)\right) \subset \mathbb{R}^{n}$. We define

$$
\nu_{s, \infty}(u):=\sup _{j}\left\|\left(\eta_{j} u\right) \circ \phi_{j}^{-1}\right\|_{W^{s, \infty}(B)}
$$

and, for $1 \leq p<\infty$,

$$
\nu_{s, p}(u)^{p}:=\sum_{j}\left\|\left(\eta_{j} u\right) \circ \phi_{j}^{-1}\right\|_{W^{s, p}(B)}^{p} .
$$

Let either $s \geq 0$ or $1<p<\infty$. Then $u \in W^{s, p}(M)$ if, and only if, $\nu_{s, p}(u)<\infty$. Moreover, $\nu_{s, p}(u)$ defines an equivalent norm on $W^{s, p}(M)$. A similar results holds for the spaces $W^{s, p}(M ; E)$.

The proof is a consequence of the following elementary and well-known lemma, which will be used also later in the paper. We state it for $E$.

Lemma 3.3. Let $\psi_{j}: M \rightarrow E, k \in \mathbb{Z}_{+}$, and $1 \leq p \leq \infty$. Assume that there exists an integer $\kappa$ such that a point $x \in M$ can belong to no more than $\kappa$ of the sets $A_{j}=\operatorname{supp}\left(\psi_{j}\right)$. Let $f=\sum_{j} \psi_{j}$. Then

$$
\|f\|_{W^{k, p}(M ; E)} \leq \kappa\left(\sum_{j}\left\|\psi_{j}\right\|_{W^{k, p}(M ; E)}^{p}\right)^{1 / p} .
$$

For $p=\infty$, we replace the right hand side with $\max \left\|\psi_{j}\right\|_{W^{k, \infty}(M)}$.

This lemma leads to the following standard mapping property (see for example $[28,41,42])$.

Proposition 3.4. Let $T: \mathcal{C}_{c}^{\infty}(M ; E) \rightarrow \mathcal{C}_{c}^{\infty}(M ; E)$ be a pseudodifferential operator of order $r$ on $M$ with distribution kernel supported in $\{d(x, y) \leq 2 a\}$. Assume that the trivializations $T_{\phi}$ (with $\phi=\phi_{\xi \eta}$ as in (4)) form a bounded family of pseudodifferential operators on $\mathbb{R}^{n}$. Then $T$ defines a continuous map $W^{s, p}(M ; E) \rightarrow$ $W^{s-r, p}(M ; E)$ for any $1<p<\infty$.

We are ready now to prove the following important technical result. Our result is slightly more precise than that in $[41,42]$.

Theorem 3.5. Let $1<p<\infty$. Let $\mu>K$, where $K$ is the constant appearing in Equation (14). For any any uniformly elliptic operator $L: \mathcal{C}_{c}^{\infty}(M ; E) \rightarrow \mathcal{C}_{c}^{\infty}(M ; E)$ satisfying Assumptions 1, 2, and 3, the operator $\left(\mu^{2}+L\right)^{s}$ extends to a continuous isomorphism

$$
\left(\mu^{2}+L\right)^{s}: W^{r, p}(M ; E) \rightarrow W^{r-2 s, p}(M ; E) .
$$

for $r \in \mathbb{R}$.

Proof. Let $f(x)=\left(\mu^{2}+x^{2}\right)^{s}$ and let $f=f_{1}+f_{2}$ be the finite propagation speed decomposition of $f$. On the one hand, Theorem 2.8 (i) and Proposition 3.4 show that $f(\sqrt{L})$ maps $W^{r}(M) \rightarrow W^{r-2 s}(M)$ continuously. On the other hand, Theorem 2.8 (ii) and Corollary 2.13 show that, for $\mu>K, f_{2}(\sqrt{L})$ also maps $W^{r}(M) \rightarrow W^{r-2 s}(M)$ continuously. We obtain that $\left(\mu^{2}+L\right)^{s}:=f(\sqrt{L})$ maps $W^{r}(M) \rightarrow W^{r-2 s}(M)$ continuously. 
Since $\left(\mu^{2}+L\right)^{s}\left(\mu^{2}+L\right)^{t} \phi=\left(\mu^{2}+L\right)^{s+t} \phi$ for $\phi \in H^{\infty}(M)=\cap_{m} H^{m}(M)$ and any $s, t \in \mathbb{R}$ by Corollary 1.7 , we obtain $\left(\mu^{2}+L\right)^{s}\left(\mu^{2}+L\right)^{t}=\left(\mu^{2}+L\right)^{s+t}$ on $W^{t, p}(M)$ for any $t \in \mathbb{R}$ and $1<p<\infty$ by the desnity of $\mathcal{C}_{\mathrm{c}}^{\infty}(M)$ in $W^{t, p}(M)$. In particular $\left(\mu^{2}+L\right)^{s}$ is invertible with inverse $\left(\mu^{2}+L\right)^{-s}$.

Proceeding as in the proof of Proposition 2.12, we obtain the following corollaries. We continue to denote by $\Xi_{\theta}$ the sector defined in Equation (13).

Corollary 3.6. Let $1<p \leq q<\infty, s \leq r$, and $\theta>0$. For $t \in \Xi_{\theta}$, let $f(z)=e^{-t z^{2}}$, and let $f=f_{1}+f_{2}$ be the finite propagation speed decomposition of $f$, Equation (12). Then, for any $N, f_{2}(\sqrt{L})$ defines a bounded map $f_{2}(\sqrt{L})$ : $W^{s, p}(M ; E) \rightarrow W^{r, q}(M ; E)$ with norm $\leq C|t|^{N}$, where $C>0$ may depend on $N$, $p, q, r, s$, and the structural constants, but not on $t \in \Xi_{\theta}$. If $s \geq 0$ and $r \in \mathbb{Z}_{+}$, we allow $1 \leq p \leq q \leq \infty$.

Proof. If $s \geq 0$, we can just replace $W^{s, p}(M ; E) \subset L^{p}(M ; E)$ with $L^{p}(M ; E)$ without affecting continuity and the norm estimates. The same argument shows that we can replace $W^{r, q}(M ; E)$ with $W^{2 k, q}(M ; E) \supset W^{r, q}(M ; E)$, if $r \leq 2 k$, again without affecting continuity and the norm estimates. Also, by Theorem 3.5 and the identity $(\mu+L)^{s / 2} f_{2}(\sqrt{L})=f_{2}(\sqrt{L})(\mu+L)^{s / 2}$, we can replace $s<0$ with $s=0$ when $1<p \leq q<\infty$.

Thus, we can assume that $s=0$ and that $r=2 k, k \in \mathbb{Z}_{+}$. We shall prove that $(\mu+L)^{k} f_{2}(\sqrt{L})$ maps $L^{p}(M ; E) \rightarrow L^{q}(M ; E)$ with norm $\leq C|t|^{N}$, for $1 \leq p \leq q \leq$ $\infty$. The volume estimate of Equation (14) together with the estimate of Corollary 2.6 , for $\tau$ large, shows that, for each fixed $N$, the integrals

$$
|t|^{-N} \int_{M}\left|(\mu+L)^{k} f_{2}(\sqrt{L})(x, y)\right| d x \quad \text { and } \quad|t|^{-N} \int_{M}\left|(\mu+L)^{k} f_{2}(\sqrt{L})(x, y)\right| d y
$$

are uniformly bounded in $x, y$, and $t \in \Xi_{\theta}$. An application of the Riesz lemma then proves the result for $p=q$. The norm of $(\mu+L)^{k} f_{2}(\sqrt{L})$ as a map $L^{1}(M ; E) \rightarrow$ $L^{\infty}(M ; E)$ is bounded by $\left\|(\mu+L)^{k} f_{2}(\sqrt{L})(x, y)\right\|_{L^{\infty}} \leq C|t|^{N}$. This gives the result for arbitrary $p \leq q$, by complex interpolation. (For the case $q=\infty$, we also need to prove analogous estimates for $P f_{2}(\sqrt{L})$, where $P$ is a differential operator of order $\leq 2 k$ with uniformly bounded coefficients.)

Next, we want to establish norm bounds on the operator $e^{-t L}$ uniform in $t$. Therefore, we have to assume that $t$ is bounded away from 0 . In fact, the case $t=1$ suffices.

Corollary 3.7. Let $1<p \leq q<\infty$ and $s \leq r$ as in Corollary 3.6. Let $f=e^{-x^{2}}$ and let again $f=f_{1}+f_{2}$ be its finite propagation speed decomposition. Then the norms of the operators

$$
e^{-L}, f_{1}(\sqrt{L}): W^{s, p}(M ; E) \rightarrow W^{r, q}(M ; E)
$$

are bounded by a constant that may depend on $p, q, r, s$, and the structural constants, but is independent of $M$ and $L$. If $s \geq 0$, we allow $1 \leq p \leq q \leq \infty$.

Proof. We proceed as in the proof of the Corollary 3.6. Again, we can assume $s=0$ and $r=2 k, k \in \mathbb{Z}_{+}$. Therefore we need to prove that $(\mu+L)^{k} e^{-L}$ and $(\mu+L)^{k} f_{1}(\sqrt{L})$ map $L^{p}(M ; E) \rightarrow L^{q}(M ; E)$ with a norm $\leq C t^{N}$, for $1 \leq p \leq$ $q \leq \infty$. It is enough to prove it for $f_{1}(\sqrt{L})$. Indeed, we know from Corollary 
3.6 that $(\mu+L)^{k} f_{2}(\sqrt{L})$ maps $L^{p}(M ; E) \rightarrow L^{q}(M ; E)$ with a norm $\leq C t^{N}$, for $1 \leq p \leq q \leq \infty$. Therefore the same holds also for $e^{-L}=f_{1}(\sqrt{L})+f_{2}(\sqrt{L})$.

The distribution kernel of $(\mu+L)^{k} f_{1}(\sqrt{L})$ is bounded by a constant independent of $M$ or $L$, by Theorem 2.3. This observation proves the result for $p=1$ and $q=\infty$. Since the support of $f_{1}(\sqrt{L})(x, y)$ is contained in $\{d(x, y) \leq 2 a\}$, by Proposition 2.1 we obtain that $\int_{M}\left|(\mu+L)^{k} f_{1}(\sqrt{L})(x, y)\right| d y$ is bounded in $x$ by a constant that depends only on the structural constants. The integrals $\int_{M}\left|(\mu+L)^{k} f_{1}(\sqrt{L})(x, y)\right| d x$ will satisfy a similar bound. Therefore, Riesz Lemma (as in the proof of Proposition $2.12)$ shows that the norm of $(\mu+L)^{k} f_{1}(\sqrt{L})$ acting on $L^{p}(M ; E)$ is bounded by a constant depending only on the structural constants. Hence, the result holds for $(\mu+L)^{k} f_{1}(\sqrt{L})(x, y)$ and $p=q$. The case of arbitrary $p \leq q$ follows by complex interpolation.

Remark 3.8. We can replace $e^{-L}$ with $e^{-t L}$ above, as long as $t \geq \epsilon>0$, is bounded away from 0 . The constants will then depend on $\epsilon$ as well. The dependence on $\epsilon$ will be made explicit in Corollary 3.12 and Theorem 3.13 in the next section.

3.1. Estimates on $\mathbb{R}^{n}$. In this section, we consider the particular case $M=\mathbb{R}^{n}$. We shall denote by $\alpha_{t}: L^{p}\left(\mathbb{R}^{n}\right) \rightarrow L^{p}\left(\mathbb{R}^{n}\right)$ the continuous bijection

$$
\alpha_{t}(f)(x)=f(t x), \quad t>0 .
$$

In particular, $t^{n / p} \alpha_{t}$ is an isometry of $L^{p}\left(\mathbb{R}^{n}\right)$ and hence $\left\|\alpha_{t}\right\|=t^{-n / p}$ on this space. More generally, on Sobolev spaces, we can conclude the following.

Lemma 3.9. For $t \in(0, \infty), \alpha_{t}$ is a bounded linear map from $W^{s, p}(M), s \geq 0$, to itself with norm $\leq C t^{-n / p}(1+t)^{s}$, with $C$ independent of $t$.

Proof. This is checked directly for $s \in \mathbb{Z}_{+}$. For $s \in \mathbb{R}_{+}$, the result follows by interpolation.

We let $\alpha_{t}$ act on each component on $L^{p}\left(\mathbb{R}^{n} ; E\right)=L^{p}\left(\mathbb{R}^{n}\right)^{\mu}$, when $E \rightarrow \mathbb{R}^{n}$ is the trivial bundle $\mathbb{C}^{\mu} \rightarrow \mathbb{R}^{n}$. If $L$ is a differential operator $L=\sum_{|\alpha| \leq 2} a_{\alpha}(x) \partial^{\alpha}$, we define $L_{t}$ by the formula

$$
L_{t}:=t\left[\alpha_{t^{1 / 2}} \circ L \circ \alpha_{t^{-1 / 2}}\right]=\sum_{|\alpha| \leq 2} t^{1-|\alpha| / 2} a_{\alpha}\left(t^{1 / 2} x\right) \partial^{\alpha} .
$$

In particular, if $L$ satisfies Assumptions 1, 2, and 3, then $L_{t}$, for $0<t \leq 1$, will also satisfy these assumptions (with the same structural constants). Recall from Corollary 3.7 that $e^{-t L}$ maps $L^{p}\left(\mathbb{R}^{n} ; E\right)$ to itself.

Lemma 3.10. We have $e^{-t L}=\alpha_{t^{-1 / 2}} \circ e^{-L_{t}} \circ \alpha_{t^{1 / 2}}$ on $L^{p}\left(\mathbb{R}^{n} ; E\right)$.

Proof. Consider first the case $p=2$. Then $t^{n / 2} \alpha_{t}$ is a unitary map for all $t>0$, and the result follows from the unitary invariance of the functional calculus with continuous functions together with the relation $t L=\alpha_{t^{-1 / 2}} \circ L_{t} \circ \alpha_{t^{1 / 2}}$. For arbitrary $p<\infty$, we exploit the density of $\mathcal{C}_{\mathrm{c}}^{\infty}\left(\mathbb{R}^{n} ; E\right)$ in $L^{p}\left(\mathbb{R}^{n} ; E\right)$ and the fact that $e^{-L_{t}}$ is a bounded operator on $L^{p}\left(\mathbb{R}^{n} ; E\right.$ ) (Corollary 3.7). For $p=\infty$, we also use the duality $L^{\infty}=\left(L^{1}\right)^{*}$.

The following theorem establishes the needed mapping properties of the heat kernel on $\mathbb{R}^{n}$. Although this result has been stated in the literature, we prove it here in a form that is suitable for our applications. One of the main points is that our estimates are uniform in $L$, as long as Assumptions 1-3 are satisfied. 
Theorem 3.11. Let $1<p \leq q<\infty$ and $s \leq r$, and $2 \gamma=n\left(\frac{1}{p}-\frac{1}{q}\right)+(r-s)$. Then $e^{-t L}$ maps $W^{s, p}\left(\mathbb{R}^{n} ; E\right)$ continuously to $W^{r, q}\left(\mathbb{R}^{n} ; E\right)$ with norm $\left\|e^{-t L}\right\| \leq C t^{-\gamma}$. The constant $C$ may depend on $r$, on $p$, and on the structural constants, but is independent of $t \in(0,1]$. If $s=0$ and $r \geq 0$, then we allow $1 \leq p \leq q \leq \infty$.

Proof. We first reduce to the case $s=0$. Indeed, using Theorem 3.5, we can write $e^{-t L}: W^{s, p}\left(\mathbb{R}^{n} ; E\right) \rightarrow W^{r, q}\left(\mathbb{R}^{n} ; E\right)$ as the composition

$$
W^{s, p}\left(\mathbb{R}^{n} ; E\right) \stackrel{(\mu+L)^{s / 2}}{\longrightarrow} L^{p}\left(\mathbb{R}^{n} ; E\right) \stackrel{e^{-t L}}{\longrightarrow} W^{r-s, q}\left(\mathbb{R}^{n} ; E\right) \stackrel{(\mu+L)^{-s / 2}}{\longrightarrow} W^{r, q}\left(\mathbb{R}^{n} ; E\right)
$$

with $\mu \geq(K+1)^{2}$. For $p, q$, and $r$ fixed, the norms of the operators $(\mu+L)^{s / 2}$ and $(\mu+L)^{-s / 2}$ in the above composition are bounded by some constants depending only on the structural constants. Therefore, it is enough to prove that the norm of $e^{-t L}: L^{p}\left(\mathbb{R}^{n} ; E\right) \rightarrow W^{r-s, q}\left(\mathbb{R}^{n} ; E\right)$ is bounded by $C t^{\gamma}$. That is, we may assume that $s=0$.

The family $L_{t}$ of Equation (17) satisfies Assumptions 1, 2, and 3 for $0<t \leq 1$, so the norm of $e^{-L_{t}}: L^{p}\left(\mathbb{R}^{n} ; E\right) \rightarrow W^{r, q}\left(\mathbb{R}^{n} ; E\right)$ is uniformly bounded in $t \in(0,1]$, by Corollary 3.7. Let $C_{0}$ be a bound for these norms. Then Lemma 3.10 gives that the norm of $e^{-t L}=\alpha_{t^{-1 / 2}} \circ e^{-L_{t}} \circ \alpha_{t^{1 / 2}}: L^{p}\left(\mathbb{R}^{n} ; E\right) \rightarrow W^{r, q}\left(\mathbb{R}^{n} ; E\right)$ is

$$
\left\|e^{-t L}\right\| \leq t^{-\frac{n}{2 p}} C_{0} t^{\frac{n}{2 q}}\left(1+t^{-1 / 2}\right)^{r} \leq C t^{-\gamma}, \quad t \in(0,1] .
$$

The proof is now complete.

In the next section, we will use localization and the above estimates on $\mathbb{R}^{n}$ to establish mapping properties of heat operators for general $L^{p}$-Sobolev spaces on $M$. In order to do so, we need uniform bounds on the "local part", $f_{1}(\sqrt{L})$, in the finite spped decomposition of $e^{t L}$.

Corollary 3.12. Let $1<p \leq q<\infty$ and $0 \leq s \leq r$. Let $f=e^{-t x^{2}}$, for $t \in(0,1]$, and let $f=f_{1}+f_{2}$ be its finite propagation speed decomposition, Equation (12). Then

$$
f_{1}(\sqrt{L}): W^{s, p}\left(\mathbb{R}^{n} ; E\right) \rightarrow W^{r, q}\left(R R^{n} ; E\right),
$$

has norm $\left\|f_{1}(\sqrt{L})\right\| \leq C t^{-\gamma}$, where $2 \gamma=n\left(\frac{1}{p}-\frac{1}{q}\right)+(r-s)$ and $C>0$ is a constant that may depend on $p, q, r, s$, and the structural constants, but not on $L$. If $s=0$ and $r \geq 0$, then we allow $1 \leq p \leq q \leq \infty$.

Proof. We have that the norm of $f_{2}(\sqrt{L})=e^{-t L}-f_{1}(\sqrt{L})$ is uniformly bounded in $t \in(0,1]$ between any Sobolev spaces by Corollary 3.6.

3.2. Mapping estimates for general $M$. We now combine the results of the previous sections to prove mapping properties of $e^{-t L}$ acting between suitable Sobolev spaces.

We start by recalling a fact about sequences of numbers, which we will use in the proof. If $a_{k} \in \mathbb{C}$ is such that $\sum_{k=1}^{\infty}\left|a_{k}\right|<\infty$, then, for any $\gamma \geq 1$, we have

$$
\sum_{k=1}^{\infty}\left|a_{k}\right|^{\gamma} \leq\left(\max \left|a_{k}\right|\right)^{\gamma-1} \sum_{k=1}^{\infty}\left|a_{k}\right| \leq\left(\sum_{k=1}^{\infty}\left|a_{k}\right|\right)^{\gamma} .
$$

We are ready now to prove one of our main results. 
Theorem 3.13. Let $1<p \leq q<\infty$ and $s \leq r$. If $s=0$ and $r \in \mathbb{Z}_{+}$, then we allow $1 \leq p \leq q \leq \infty$. Then $e^{-t L}$ defines a continuous map

$$
e^{-t L}: W^{s, p}(M ; E) \rightarrow W^{r, q}(M ; E), \quad t \in(0,1],
$$

with norm $\left\|e^{-t L}\right\| \leq C t^{-\gamma}$, where $2 \gamma=n\left(\frac{1}{p}-\frac{1}{q}\right)-(r-s)$ and $C>0$ is a constant that may depend on $p, q, s, r$, and the structural constants, but is independent of $M$ and the semi-definite, uniformly elliptic $L$ (as long as the Assumptions 1, 2, and 3 are satisfied). If $s=0$ and $r \geq 0$, then we may allow $1 \leq p \leq q \leq \infty$.

Proof. Let $f=e^{-t x^{2}}=f_{1}+f_{2}$ be the finite propagation speed decomposition of $f$, Equation (12). Using the same argument based on Corollary 3.6 as in the proof of Corollary 3.12, we see that it is enough to prove that

$$
f_{1}(\sqrt{L}): W^{s, p}(M ; E) \rightarrow W^{r, q}(M ; E), \quad t \in(0,1],
$$

has norm $\left\|f_{1}(\sqrt{L})\right\| \leq C t^{-\gamma}$, where $C>0$ is as in the statement of the theorem. We assume that we have chosen $\chi$ with support in $\left[-2 a / c_{M}, 2 a / c_{M}\right]$ such that $2 a<\epsilon$, where $\epsilon$ is the constant in Lemma 3.1 and $a$ is one of the structural constants.

We shall use the alternative definition of the norm in Lemma 3.2. We proceed as in the proof of Proposition 3.4. Let $\eta_{j}$ be a partition of unity as in Lemma 3.1, in particular, the support of $\eta_{j}$ is contained in the ball $B_{a}\left(x_{j}\right)$ and no more than $\kappa$ of the balls $B_{3 a}\left(x_{j}\right)$ have a non-empty intersection. Theorem 2.5 then guarantees that $f_{1}(\sqrt{L})\left(\eta_{j} u\right)$ has support in $B_{3 a}\left(x_{j}\right)$.

Let $\psi_{j}$ be the normal coordinate trivialization of $E$ over $B_{7 a}\left(x_{j}\right)$. Let $L_{j}=L_{\phi_{j}}$ be the operator on $\mathcal{C}_{\mathrm{c}}^{\infty}\left(\mathbb{R}^{n} ; E\right.$ ) defined by $L$ using the trivializations $\phi_{j}$ (as in Assumption 2 or in Theorem 2.5), for instance). Finally, let $u \in W^{s, p}(M ; E)$ and set $u_{k}$ to be the image of $\eta_{k} u$ under the trivialization $\phi_{k}$.

Assume first that $q<\infty$. Then Lemma 3.3, the uniform bound on the derivatives of the trivializations $\phi_{j}$ and their inverses, Corollary 3.12, Equation (5), and, for the last inequality, Lemma 3.2 give

$$
\begin{aligned}
\left\|f_{1}(\sqrt{L}) u\right\|_{W^{r, q}}^{q}=\left\|\sum_{j} f_{1}(\sqrt{L})\left(\eta_{j} u\right)\right\|_{W^{r, q}}^{q} \leq \kappa^{q} \sum_{j}\left\|f_{1}(\sqrt{L})\left(\eta_{j} u\right)\right\|_{W^{r, q}}^{q} \\
\leq C \kappa^{q} \sum_{j}\left\|f_{1}\left(\sqrt{L_{j}}\right) u_{j}\right\|_{W^{r, q}}^{q} \leq C t^{-\gamma q} \sum_{j}\left\|u_{j}\right\|_{W^{s, p}}^{q} \leq C t^{-\gamma q} \sum_{j}\left\|\eta_{j} u\right\|_{W^{s, p}}^{q} \\
\leq C t^{-\gamma q}\left(\sum_{j}\left\|\eta_{j} u\right\|_{W^{s, p}}^{p}\right)^{q / p} \leq C t^{-\gamma q}\|u\|_{W^{s, p}}^{q},
\end{aligned}
$$

where we have absorbed $\kappa^{p}$ into $C$.

For $q=\infty$ and $s \in \mathbb{Z}_{+}$, we have instead

$$
\begin{aligned}
& \left\|f_{1}(\sqrt{L}) u\right\|_{W^{r, \infty}}=\left\|\sum_{j} f_{1}(\sqrt{L})\left(\eta_{j} u\right)\right\|_{W^{r, \infty}} \leq \kappa \max _{j}\left\|f_{1}(\sqrt{L})\left(\eta_{j} u\right)\right\|_{W^{r, \infty}} \\
& \leq C \kappa \max _{j}\left\|f_{1}\left(\sqrt{L_{j}}\right) u_{j}\right\|_{W^{r, \infty}} \leq C t^{-\gamma} \max _{j}\left\|u_{j}\right\|_{L^{p}} \leq C t^{-\gamma}\left(\sum_{j}\left\|\eta_{j} u\right\|_{L^{p}}^{p}\right)^{1 / p} \\
& \leq C t^{-\gamma}\|u\|_{L^{p}} .
\end{aligned}
$$

The proof is now complete.

A similar argument gives that $L$ generates a holomorphic semigroup on $L^{p}(M ; E)$ for any $1 \leq p<\infty$. 
Theorem 3.14. Let $1 \leq p<\infty$. Then $e^{-t L} \in \mathcal{L}\left(L^{p}(M ; E)\right)$ is a holomorphic function defined for $\Re(t)>0$ and $e^{-t L} e^{-s L}=e^{-(t+s) L}$ for $\Re(t), \Re(s)>0$. Moreover, $\left\|e^{-t L} u-u\right\|_{L^{p}} \rightarrow 0$ as $t \rightarrow 0$ and $t \in \Xi_{\theta}$, where $\Xi_{\theta}$ is the sector defined by Equation (13).

Proof. The first part follows from Proposition 2.14 since the map $\{\Re(t)>0\} \ni$ $t \rightarrow e^{-t x^{2}} \in \mathcal{F}_{\tau}^{N}$ is holomorphic for any fixed $N \in \mathbb{Z}_{+}$and $\tau>0$. The equation $e^{-t L} e^{-s L}=e^{-(t+s) L}$ is verified for $p=2$ by functional calculus. For $p<\infty$ it follows from the density of $\mathcal{C}_{\mathrm{c}}^{\infty}(M ; E)$ in $L^{p}(M ; E)$.

For the second part, let $f_{t}(x)=e^{-t x^{2}}$ and $f_{t}=f_{t 1}+f_{t 2}$ the finite propagation speed decomposition of $f_{t}$. Corollary 2.7 and Proposition 2.14 give that $f_{t 2}(\sqrt{L}) \in$ $\mathcal{L}\left(L^{p}(M ; E)\right)$ and $\left\|f_{t 2}(\sqrt{L})\right\|_{L^{p}} \rightarrow 0$ as $t \rightarrow 0, t \in \Xi_{\theta}$. Hence, it is enough to prove that $\left\|f_{t 2}(\sqrt{L}) u-u\right\|_{L^{p}} \rightarrow 0$ for any $u \in L^{p}(M ; E)$. To this end, we proceed as in the proof of Theorem 3.13. We shall use the notation introduced in that theorem. We observe that the result holds if $M=\mathbb{R}^{n}$ (see, e.g., [8, 19, 31]). Moreover, we know that $f_{t 1}\left(\sqrt{L_{j}}\right)$ is uniformly bounded in $\mathcal{L}\left(L^{p}\left(\mathbb{R}^{n} ; \mathbb{C}^{\mu}\right)\right)=M_{\mu}\left(\mathcal{L}\left(L^{p}\left(\mathbb{R}^{n}\right)\right)\right)$, in view of Corollary 3.12.

Then, again Lemma 3.3, the uniform bounds on the derivatives of the trivializations $\phi_{j}$ and their inverses, Corollary 3.12, Equation (5), and, for the last inequality, Lemma 3.2 then give

$$
\begin{aligned}
\| f_{t 1}(\sqrt{L}) u & -u\left\|_{L^{p}}^{p}=\right\| \sum_{j}\left(f_{t 1}(\sqrt{L})\left(\eta_{j} u\right)-\eta_{j} u\right) \|_{L^{p}}^{p} \\
& \leq \kappa^{q} \sum_{j}\left\|f_{t 1}(\sqrt{L})\left(\eta_{j} u\right)-\eta_{j} u\right\|_{L^{p}}^{p} \leq C \kappa^{p} \sum_{j}\left\|f_{t 1}\left(\sqrt{L_{j}}\right) u_{j}-u_{j}\right\|_{L^{p}}^{p} .
\end{aligned}
$$

Since $\left\|f_{t 1}\left(\sqrt{L_{j}}\right) u_{j}-u_{j}\right\|_{L^{p}} \leq C\left\|u_{j}\right\|,\left\|f_{t 1}\left(\sqrt{L_{j}}\right) u_{j}-u_{j}\right\|_{L^{p}} \rightarrow 0$ as $t \rightarrow 0, t \in \Xi_{\theta}$, and $\sum\left\|u_{j}\right\|_{L^{p}}^{p} \leq C\|u\|_{L^{p}}^{p}$, an application of the Lebesgue dominated convergence theorem gives the result.

\section{Maximal $L^{p}-L^{q}-$ Regularity}

We now extend our last results to prove that the second order operator $L$ satisfying Assumptions 1-3 also satisfies "maximal $L^{p}-L^{q}-$ regularity."

If $X$ is a Banach space and $1 \leq p<\infty$, we denote by $L^{p}(0, b, X)$ the completion of $\mathcal{C}([0, a] ; X)$ in the norm $\|f\|_{L^{p} X}^{p}=\int_{0}^{a}\|f(t)\|_{X}^{p}$. (We have denoted by $\mathcal{C}([0, a] ; X)$ the space of continuous functions $[0, a] \rightarrow X$.) For simplicity, we also write $L^{p} X:=$ $L^{p}(0,1, X)$. In what follows, we shall consider only $X=L^{q}(M ; E)$ and $X=$ $W^{2, q}(M ; E), 1<q<\infty$. In fact, for simplicity of notation, we consider explicitely only the scalar case (i.e., $E=M \times \mathbb{C}$ ). The general case is completely similar.

Definition 4.1. Let $k(t, x, y)$ be a measurable function defined on $\mathbb{R} \times M \times M$ that vanishes for $t \leq 0$. We say that $k$ satisfies maximal $L^{p}-L^{q}$ regularity if

$$
F(t, x):=\int_{0}^{t} \int_{M} k\left(t-s, x, x^{\prime}\right) f\left(s, x^{\prime}\right) d x^{\prime} d s
$$

is in $L^{p}\left(0, b, W^{2, q}(M)\right)$ for any $f \in L^{p}\left(0, b, L^{q}(M)\right)$ and there exists a constant $C$ independent of $f$ such that $\|F\|_{L^{p} W^{2, q}} \leq C\|f\|_{L^{p} L^{q}}$. The least $C$ with this property is denoted $\|k\|_{M R}$. 
We say that a second-order differential operator $L$ (fulfilling Assumptions 1,2,3) satisfies maximal $L^{p}-L^{q}$ regularity if $k\left(t, x, x^{\prime}\right):=e^{-t L}\left(x, x^{\prime}\right)$ satisfies maximal $L^{p_{-}}$ $L^{q}$ regularity.

We shall denote $F$ above by $k * f$. Maximal $L^{p}-L^{q}$ regularity thus means that $f \rightarrow k * f$ defines a continuous map $L^{p}\left(0, b, L^{q}(M)\right) \rightarrow L^{p}\left(0, b, W^{2, q}(M)\right)$. Then $\|k\|_{M R}$ is the norm of this map. It is known that if $k=e^{-t L}$, the maximal $L^{p}-L^{q}$ regularity is independent of $b>0$ and $1<p<\infty$ [21, 43, 2]. (See also [22] for a related result on the independence of the spectrum on $p$.)

We introduce the space

$$
X_{p, q}:=\left\{f \in L^{p}\left(0, b, W^{2, q}(M)\right), f^{\prime} \in L^{p}\left(0, b, L^{q}(M)\right), f(0)=0\right\},
$$

with the induced norm that makes it a Banach space. The main reason we are interested in maximal regularity, is that $L$ satisfies maximal $L^{p}-L^{q}$ regularity if, and only if,

$$
\partial_{t}+L: X_{p, q} \rightarrow L^{p}\left(0, b, L^{q}(M)\right)
$$

is an isomorphism of Banach spaces. Then there is a constant $C_{p, q}>0$ such that

$$
C_{p, q}^{-1}\left\|e^{-t T}\right\|_{M R} \leq\left\|\left(\partial_{t}+L\right)^{-1}\right\| \leq C_{p, q}\left\|e^{-t T}\right\|_{M R} .
$$

The constant $C_{p, q}$ depends only on $p, q, b$, and the structural constants.

We shall prove that indeed our operator $L$ satisfies maximal $L^{p}-L^{q}$ regularity. This is the content of Theorem 4.4, the main result of this section. We first prove some preliminary lemmas, in order to exploit the results of the previous sections.

Let $f_{t}=e^{-t x^{2}}=f_{t 1}+f_{2 t}$ be the finite propagation speed decomposition of $f_{t}$, $t>0$.

Lemma 4.2. The kernel $\tilde{k}\left(t, x, x^{\prime}\right)=f_{t 2}\left(t, x, x^{\prime}\right)$ satisfies maximal $L^{p}-L^{q}$ regularity with $\|\cdot\|_{M R^{-}}$norm depending only on the structural constants. We have that $L$ satisfies maximal $L^{p}-L^{q}$ regularity if, and only if, $k\left(t, x, x^{\prime}\right)=f_{t 1}\left(t, x, x^{\prime}\right)$ satisfies maximal $L^{p}-L^{q}$ regularity.

Proof. The first part follows from the observation that $t \rightarrow f_{t 2}(\sqrt{L})$ is continuous as a map from $[0,1]$ to $\mathcal{L}\left(L^{q}(M), W^{2, q}(M)\right)$, which is a consequence of Corollary 3.6 and Proposition 2.14 applied to the functions $\left(1+x^{2}\right) f_{t 2}(x)$.

The second part follows from the fact that the sum of two kernels satisfying maximal $L^{p}-L^{q}$ regularity also satisfies maximal $L^{p}-L^{q}$ regularity.

Again, in the next lemma the main point is that we obtain estimates independent of the operator $L$ satisfying Assumptions 1-3.

Lemma 4.3. Assume that $M=S^{n}$. Assume that the second order differential operator $L$ satisfies Assumptions 1-3. Then $L$ satisfies maximal $L^{p}-L^{q}$ regularity with $\|\cdot\|_{M R^{-}}$norm depending only on the structural constants (thus independent of L satisfying Assumptions 1-3).

Proof. For $M=S^{n}$ it is known that $L$ satisfies maximal $L^{p}-L^{q}$ regularity whenever $L$ satisfies Assumptions 1-3, see $[21,31]$. We only need to prove that the $\|\cdot\|_{M R^{-}}$ norm is independent of $L$. In view of Equation (19), it is enough to prove that $\left\|\left(\partial_{t}+L\right)^{-1}\right\|$ is bounded in $L$ satisfying Assumptions $1-3$.

Let $Y\left(S^{n}\right)$ be the space of second order differential operators on $S^{n}$ satisfying Assumptions 1-3 with the topology induced by the topology of $\mathcal{C}^{\infty}$-functions on $S^{n}$. If $L, L_{1} \in Y$ are close, then $L_{1}-L=\left(\partial_{t}+L_{1}\right)-\left(\partial_{t}+L\right): X_{p, q} \rightarrow L^{p}\left(0, b, L^{q}(M)\right)$ has 
small norm, and hence $\left\|\left(\partial_{t}+L\right)^{-1}\right\|$ is a continuous function on $Y\left(S^{n}\right)$. Therefore $\left\|\left(\partial_{t}+L\right)^{-1}\right\|$ is uniformly bounded, because $Y\left(S^{n}\right)$ is compact by the classical Arzelá-Ascoli theorem.

We are ready now to prove our "maximal regularity" result for the operator $L$.

Theorem 4.4. Let $L$ be a second order differential operator satisfying Assumptions 1-3. Then $L$ satisfies maximal $L^{p}-L^{q}$ regularity for $1<p, q<\infty$. Moreover, the norm $\left\|\left(\partial_{t}+L\right)^{-1}\right\|$ is bounded in $L$, with a bound depending only on the structural constants and $b>0$.

Proof. By Lemma 4.2, if is enough to prove that $k\left(t, x, x^{\prime}\right):=f_{t 1}(\sqrt{L})\left(x, x^{\prime}\right)$ satisfies maximal $L^{p}-L^{q}$ regularity. We know from $[21,43,2]$ that it is enough to consider the case $p=q$ and $b=1$. In that case, $L^{p} L^{p}(M)=L^{p}([0,1] \times M)$.

We then use the arguments and notation of the proof of Theorem 3.13. (The partition of unity $\eta_{j}$ is extended to $[0,1] \times M$ to be independent of $s \in[0,1]$ and the operators $L_{j}$ are extensions to $S^{n}$ of the localizations of $L$ to the support of $\left.\eta_{j}.\right)$ Let $f_{t 1}(\sqrt{L}) * u(t, x):=\int_{0}^{t} \int_{M} f_{(t-s) 1}(\sqrt{L})\left(x, x^{\prime}\right) u\left(s, x^{\prime}\right) d x^{\prime} d s$. Then

$$
\begin{aligned}
& \left\|f_{t 1}(\sqrt{L}) * u\right\|_{L^{p} W^{2, p}(M)}^{p}=\left\|\sum_{j} f_{t 1}(\sqrt{L}) *\left(\eta_{j} u\right)\right\|_{L^{p} W^{2, p}(M)}^{p} \\
& \leq \kappa^{p} \sum_{j}\left\|f_{t 1}(\sqrt{L}) *\left(\eta_{j} u\right)\right\|_{L^{p} W^{2, p}(M)}^{p} \leq C \kappa^{p} \sum_{j}\left\|f_{t 1}\left(\sqrt{L_{j}}\right) * u_{j}\right\|_{L^{p} W^{2, p}\left(S^{n}\right)}^{p} \\
& \leq C \sum_{j}\left\|u_{j}\right\|_{L^{p} L^{p}\left(S^{n}\right)} \leq C \sum_{j}\left\|\eta_{j} u\right\|_{L^{p} L^{p}(M)}^{p} \leq C\|u\|_{L^{p} L^{p}(M)}^{p},
\end{aligned}
$$

where the third inequality is by Lemma 4.3. The last part follows from the fact that all the constants depend only on the structural constants and $b>0$.

From the maximal $L^{p}-L^{q}$ regularity we obtain a well-posedness result for parabolic equations with variable coefficients. See also [21, 30, 31, 38] for similar results and other applications of maximal regularity. Let

$$
Y_{p, q}=\left[W^{2, q}(M ; E), L^{q}(M ; E)\right]_{1 / p, p}
$$

be the interpolating space obtained by the "real method" (see [31], for instance).

Theorem 4.5. Let $L_{t}, t \in[0, b]$, be a smooth family of second order differential operators satisfying Assumptions 1-3. Then for any $f \in L^{p}\left(0, b, L^{q}(M ; E)\right), 1<$ $p, q<\infty$, and $g \in Y_{p, q}$, the equation

$$
\partial_{t} u(t, x)+L_{t} u(t, x)=f(t, x), \quad u(0, x)=g(x),
$$

has a unique solution $u \in X_{p, q}$ and $\|u\|_{X_{p, q}} \leq C\|f\|_{L^{p} L^{q}}$, where $C$ is a constant that depends only on the structural constants and the $W^{2, \infty}(M)$-norm of the first derivative $\partial_{t} a_{\alpha}(t, x)$ of the coefficients of $L_{t}$.

Proof. It is known that we can assume $g=0$ [21, 38]. Also, we shall assume again, for simplicity, that $E=M \times \mathbb{C}$. We shall use the method in [30].

The norm of operator $X_{p q} \ni u \rightarrow L_{t} u-L_{0} u$ obtained by replacing the family $L_{t}$ with the constant family $L_{0}$ (i.e., obtained by freezing the coefficients at $t=0$ ) can be made arbitrarily small as $b \rightarrow 0$. Moreover, the norm of

$$
\left(\partial_{t}+L_{0}\right)^{-1}: L^{p}\left(0, b, L^{q}(M)\right) \rightarrow X_{p, q}
$$


increases with $b$. Therefore the norm of the operator

$$
\left(L_{t}-L_{0}\right)\left(\partial_{t}+L_{0}\right)^{-1}: L^{p}\left(0, b, L^{q}(M)\right) \rightarrow L^{p}\left(0, b, L^{q}(M)\right)
$$

can be made as small as we want. In particular, $\left\|\left(L_{t}-L_{0}\right)\left(\partial_{t}+L_{0}\right)^{-1}\right\| \leq 1 / 2$ if $b \leq b_{0}$, where $b_{0}>0$ depends only on the structural constants and the $W^{2, \infty_{-}}$ bounds on the coefficients of $\partial_{t} L_{t}$. Therefore

$$
\partial_{t}+L_{t}=\left[1+\left(L_{t}-L_{0}\right)\left(\partial_{t}+L_{0}\right)^{-1}\right]\left(\partial_{t}+L_{0}\right): X_{p q} \rightarrow L^{p}\left(0, b, L^{q}(M)\right)
$$

is invertible for $b \leq b_{0}$.

At this point, we can use Theorem 4.4 to argue that $b_{0}$ is independent of $L_{0}$. Therefore, we can replace $L_{0}$ with $L_{b}$ and obtain that we have a solution of our initial problem on the interval $\left[0,2 b_{0}\right]$. Repeating the argument, we obtain a solution on the whole interval $[0, a]$ since restriction at $b$ defines a continuous surjection $X_{p, q} \rightarrow Y_{p, q}$.

\section{Applications to SEMi-Linear PARABOliC EQUATIONS}

In this section, we apply the results of Sections 2 and 3 to the study of the Initial Value Problem (IVP) for semilinear parabolic equations of the form (1). We begin by considering abstract evolution equations in Banach spaces, for which semigroup techniques are especially well suited. We follow [37] in our presentation.

5.1. Abstract semilinear equations. Let $L$ be an operator on a Banach space $X$ with domain $\mathcal{D}(L)$, such that $-L$ generates a $C^{0}$-semigroup $U(t), t \geq 0$, on $X$. Consider the following initial value problem

$$
\partial_{t} u+L u=F(u), \quad u(0)=f,
$$

where $F: X \rightarrow X$ is continuous and $f \in X$.

An important example is provided by the semilinear scalar heat equation on $M$

$$
\partial_{t} u=\Delta_{M} u(t, x)+h(u(t, x)),
$$

where $u:[0, T] \times M \rightarrow \mathbb{C}$, and $h: \mathbb{C} \rightarrow \mathbb{C}$ is a locally Lipschitz function satisfying

$$
|h(z)-h(w)| \leq C|z-w|\left(1+|z|^{\sigma-1}+|w|^{\sigma-1}\right),
$$

for some $\sigma>1$. Throughtout this section, we use the notation $u(t)(x)=u(t, x)$, $t \geq 0, x \in M$. Equations of this form arise in control theory and mathematical biology.

We now recall the notion of strong and mild solutions in this context [37].

Definition 5.1. Let $u:[0, T] \rightarrow X$ be a continuous function. We shall say that $u$ is a strong solution of the Initial Value Problem (20), if $u$ is differentiable almost everywhere on $[0, T], u(0)=f, u(t) \in \mathcal{D}(A)$ for $t>0$, and the Equation (20) is satisfied almost everywhere in $t>0$.

Similarly, we shall say that $u$ is a mild solution of the Initial Value Problem (20) if it satisfies the integral equation

$$
u(t)=U(t) f+\int_{0}^{t} U(t-s) F(u(s)) d s, \quad 0 \leq t \leq T,
$$

where the integral is interpreted as a (possibly improper) Bochner integral. 
In general, the integral formulation is weaker than the initial IVP, because we only need $U(t-\cdot) F(u(\cdot)) \in L^{1}([0, T], X)$. This is especially true if $U(t)$ has smoothing properties like the heat semigroup. However, if $F$ is Lipschitz, $X$ is reflexive, and $f \in \mathcal{D}(A)$, any mild solution is actually a strong solution of (20) (see again $[37])$.

Since for a given $f$, mild solutions are just fixed points of the nonlinear map $\mathfrak{N}$ defined as

$$
\mathfrak{N}(u)(t)=U(t) f+\int_{0}^{t} U(t-s) F(u(s)) d s,
$$

existence and uniqueness may be obtained by applying the contraction mapping theorem to $\mathfrak{N}$ for $u$ in some closed ball $B(0, A) \subset C([0, T], X)$, where $A>0$ depends on the size of the initial data. There is an extensive literature on the subject (see [31, 37, 48], and references therein), we follow [46] here.

We assume that there exists another Banach space $Y$ such that $X$ and $Y$ satisfy the following conditions:

(Loc.1) $U(t)$ is a $C^{0}$ semigroup on $X$,

(Loc.2) $U(t)$ maps $Y$ to $X$ continuously and there exists $\gamma \in(0,1)$ such that the operator norm satisfies

$$
\|U(t)\|_{\mathcal{L}(Y, X)} \leq C t^{-\gamma}
$$

for $t \in(0,1)$,

(Loc.3) $F: X \rightarrow Y$ is a locally Lipschitz map.

In the cases of interest here, we can also assume that $U(t)$ is uniformly bounded on $X$ for $t \in[0, \infty)$. Under these assumptions, the following theorem holds.

Theorem 5.2 ([46]). Under conditions (Loc.1-3), there exists a unique short-time mild solution $u \in C([0, T], X)$ to the Initial Value Problem (20). The time $T$ of existence of the solution can be estimated from below solely in terms of $\|f\|_{X}$.

5.2. Applications. Using the kernel estimates of Section 2, we give some applications of Theorem 5.2 to semilinear equations of the form (1). Here, $L$ is again the operator satisfying Assumptions 1-3. In particular, $-L$ generates a $C^{0}$ semigroup on $W^{s, p}, 1<p<\infty, s \in \mathbb{R}$, by Theorem 3.14 .

We consider the following type of non-linearities (in the spirit of [48, Chapter 16]):

NL.1 $F(u)=\operatorname{div} G(u)$, where $G: E \rightarrow E$ and the divergence is taken with respect to the connection $\nabla_{E}$ on $E$;

NL.2 $F(u, \nabla u)=F(u)$, where $F: E \rightarrow E$ is a smooth map that acts fiberwise, that is, for every $x \in M, F_{x}: \pi^{-1}(x) \rightarrow \pi^{-1}(x)$, with $\pi$ the projection $E \rightarrow M$, and such that $F_{x}(0)=0$. (Here, we assume $E$ to be real.)

Condition NL.1 arises, for example, in studying quasilinear hyperbolic equations by the viscosity method and includes non-linear advection. Condition NL.2 gives rise to a "reaction-diffusion" type equation.

Proposition 5.3. Let $G: E \rightarrow E$ be a locally Lipschitz map satisfying

$$
|G(u)-G(v)| \leq C|u-v|\left(1+|u|^{\sigma-1}+|v|^{\sigma-1}\right)
$$

for some $\sigma \geq 1$. Then, the IVP

$$
\partial_{t} u+L u=\operatorname{div} G(u), \quad u(0)=f,
$$


has a unique short-time solution in $C\left([0, T], L^{q}(M ; E)\right)$ for $q \geq \sigma, q>n(\sigma-1)$.

Proof. The proof is very similar to the proof of Proposition 1.3 in [48]. We sketch the main steps. We apply Theorem 5.2 with $X=L^{q}(M ; E), Y=W^{-1, q / \sigma}(M ; E)$ $(q / \sigma \geq 1)$. By Hölder's inequality:

$$
\|G(u)-G(v)\|_{L^{q / \sigma}} \leq C\left(\|u\|_{L^{q}}+\|v\|_{L^{q}}\right)^{\sigma-1}\|u-v\|_{L^{q}},
$$

so that condition (Loc.3) holds. Next, from Theorem 3.13 it follows that condition (Loc.2) is satisfied with $\gamma=n(\sigma-1) / 2 q+1 / 2$, and $\gamma<1$ if $n(\sigma-1)<q$.

Another direct application of Theorems 3.13 and 5.2 gives the following result in case NL.2.

Proposition 5.4. Let $F: E \rightarrow E$ be a smooth map, acting fiberwise on $E$ and mapping the zero section to the zero section. Then, the IVP:

$$
\partial_{t} u+L u=F(u), \quad u(0)=f,
$$

has a unique short-time solution in $C\left([0, T], W^{s, p}(M ; E)\right), s \geq 0,1<p<\infty$, provided that for any trivialization $\phi_{\xi \eta}$ of $E$ (Equation 4), the map $V: \mathbb{C}^{\mu} \rightarrow \mathbb{C}^{\mu}$ defined by

$$
V(y)=\nabla\left(\phi_{\xi \eta}\right)_{*}(F u)(x, t), \quad y=\left(\phi_{\xi \eta}\right)_{*} u(x, t),
$$

is uniformly bounded along with its first $[s]$ derivatives.

Above, $\left(\phi_{\xi \eta}\right)_{*}$ is the push-forward by the map $\phi_{\xi \eta}$.

\section{REFERENCES}

[1] A. Ashtekar and B. Krishnan. Dynamical horizons and their properties. Preprint ArXiv grqc/0308033.

[2] P. Cannarsa and V. Vespri. On maximal $L^{p}$ regularity for the abstract Cauchy problem. Boll. Un. Mat. Ital. B (6), 5(1):165-175, 1986.

[3] M. Cannone. Ondelettes, paraproduits et Navier-Stokes. Diderot Editeur, Paris, 1995. With a preface by Yves Meyer.

[4] M. Cheeger, J. Gromov and M. Taylor. Finite propagation speed, kernel estimates for functions of the Laplace operator, and the geometry of complete Riemannian manifolds. J. Differential Geom., 17(1):15-53, 1982.

[5] S. Y. Cheng, P. Li, and S. T. Yau. On the upper estimate of the heat kernel of a complete Riemannian manifold. Amer. J. Math., 103(5):1021-1063, 1981.

[6] D. Christodoulou. The action principle and partial differential equations, volume 146 of Annals of Mathematics Studies. Princeton University Press, Princeton, NJ, 2000.

[7] D. Christodoulou and S. Klainerman. The global nonlinear stability of the Minkowski space, volume 41 of Princeton Mathematical Series. Princeton University Press, Princeton, NJ, 1993.

[8] E. B. Davies. $L^{p}$ spectral independence for certain uniformly elliptic operators. In Partial differential equations and mathematical physics (Copenhagen, 1995; Lund, 1995), volume 21 of Progr. Nonlinear Differential Equations Appl., pages 122-125. Birkhäuser Boston, Boston, MA, 1996.

[9] H. Donnelly. Spectral geometry for certain noncompact Riemannian manifolds. Math. Z., 169(1):63-76, 1979.

[10] Hunsicker E. and Mazzeo R. Harmonic forms on manifolds with edges. math.DG/0503318.

[11] C. Eckart. The thermodynamics of irreversible processes. iii. relativistic theory of the simple fluid. Physical Review, 58:919-924, 1940.

[12] L.C. Evans. Partial differential equations, volume 19 of Graduate Studies in Mathematics. American Mathematical Society, Providence, RI, 1998.

[13] H. Fujita and T. Kato. On the Navier-Stokes initial value problem. I. Arch. Rational Mech. Anal., 16:269-315, 1964. 
[14] G. J. Galloway, K. Schleich, D. M. Witt, and E. Woolgar. The AdS/CFT correspondence and topological censorship. Phys. Lett. B, 505(1-4):255-262, 2001.

[15] Gregory J. Galloway. Maximum principles for null hypersurfaces and null splitting theorems. Ann. Henri Poincaré, 1(3):543-567, 2000.

[16] L. Gärding. Vecteurs analytiques dans les représentations des groups de Lie. Bull. Soc. Math. France, 88:73-93, 1960.

[17] L. Geroch, R. amd Lidblom. Dissipative relativistic fluid theories of divergence type. Physical Revew D, 41(6):1855-1861, 1990.

[18] G. Grubb. Functional calculus of pseudodifferential boundary problems, volume 65 of Progress in Mathematics. Birkhäuser Boston Inc., Boston, MA, second edition, 1996.

[19] D. Gurarie. Finite propagation speed and kernels of strictly elliptic operators. Internat. J. Math. Math. Sci., 8(1):75-91, 1985.

[20] E. Hebey. Sobolev spaces on Riemannian manifolds, volume 1635 of Lecture Notes in Mathematics. Springer-Verlag, Berlin, 1996.

[21] M. Hieber and J. Prüss. Heat kernels and maximal $L^{p}-L^{q}$ estimates for parabolic evolution equations. Comm. Partial Differential Equations, 22(9-10):1647-1669, 1997.

[22] M. Hieber and E. Schrohe. $L^{p}$ spectral independence of elliptic operators via commutator estimates. Positivity, 3(3):259-272, 1999.

[23] L. Hörmander. The analysis of linear partial differential operators. I. Classics in Mathematics. Springer-Verlag, Berlin, 2003. Distribution theory and Fourier analysis, Reprint of the second (1990) edition [Springer, Berlin; MR1065993 (91m:35001a)].

[24] T. Jeffres and P. Loya. Regularity of solutions of the heat equation on a cone. Int. Math. Res. Not., (3):161-178, 2003.

[25] Y. Kannai. Off diagonal short time asymptotics for fundamental solutions of diffusion equations. Commun. Partial Differ. Equations, 2(8):781-830, 1977.

[26] S. Klainerman and F. Nicolò. On local and global aspects of the Cauchy problem in general relativity. Classical Quantum Gravity, 16(8):R73-R157, 1999.

[27] Yu. Kordyukov. $L^{p}$-estimates for functions of elliptic operators on manifolds of bounded geometry. Russ. J. Math. Phys., 7(2):216-229, 2000.

[28] Yu.I Kordyukov. $L^{p}$-theory of elliptic differential operators on manifolds of bounded geometry. Acta Appl. Math., 23(3):223-260, 1991.

[29] J. M. Lee. Fredholm operators and Einstein metrics on conformally compact manifolds. math.DG/0105046.

[30] J.-L. Lions and E. Magenes. Non-homogeneous boundary value problems and applications. Vol. II. Springer-Verlag, New York, 1972. Translated from the French by P. Kenneth, Die Grundlehren der mathematischen Wissenschaften, Band 182.

[31] A. Lunardi. Analytic semigroups and optimal regularity in parabolic problems. Progress in Nonlinear Differential Equations and their Applications, 16. Birkhäuser Verlag, Basel, 1995.

[32] J. Maldacena. The large $N$ limit of superconformal field theories and supergravity. Adv. Theor. Math. Phys., 2(2):231-252, 1998.

[33] R.B. Melrose. Geometric scattering theory. Stanford Lectures. Cambridge University Press, Cambridge, 1995.

[34] M. Mitrea and M. Taylor. Navier-Stokes equations on Lipschitz domains in Riemannian manifolds. Math. Ann., 321(4):955-987, 2001.

[35] C. B. morrey. Multiple integrals in the calculus of variations. Die Grundlehren der mathematischen Wissenschaften, Band 130. Springer-Verlag New York, Inc., New York, 1966.

[36] V. Nistor. Pseudodifferential operators on non-compact manifolds and analysis on polyhedral domains. In Spectral geometry of manifolds with boundary and decomposition of manifolds, volume 366 of Contemp. Math., pages 307-328. Amer. Math. Soc., Providence, RI, 2005.

[37] A. Pazy. Semigroups of linear operators and applications to partial differential equations, volume 44 of Applied Mathematical Sciences. Springer-Verlag, New York, 1983.

[38] J. Prüss. Evolutionary integral equations and applications, volume 87 of Monographs in Mathematics. Birkhäuser Verlag, Basel, 1993.

[39] E. Schrohe and W. Junker. Adiabatic vacuum states on general spacetime manifolds: Definition, construction, and physical properties. Preprint, 2002.

[40] E. Schrohe and B.-W. Schulze. Boundary value problems in Boutet de Monvel's algebra for manifolds with conical singularities II. In Boundary value problems, Schrödinger operators, deformation quantization, volume 8 of Math. Top. Akademie Verlag, Berlin, 1995. 
[41] M.A. Shubin. Spectral theory of elliptic operators on noncompact manifolds. Astérisque, 207:5, 35-108, 1992. Méthodes semi-classiques, Vol. 1 (Nantes, 1991).

[42] L. Skrzypczak. Mapping properties of pseudodifferential operators on manifolds with bounded geometry. J. London Math. Soc. (2), 57(3):721-738, 1998.

[43] P. E. Sobolevskiı̌. Coerciveness inequalities for abstract parabolic equations. Dokl. Akad. Nauk SSSR, 157:52-55, 1964.

[44] R. Strichartz. Multipliers on fractional Sobolev spaces. J. Math. Mech., 16:1031-1060, 1967.

[45] M. Taylor. Pseudodifferential operators, volume 34 of Princeton Mathematical Series. Princeton University Press, Princeton, N.J., 1981 xi+452.

[46] M. Taylor. Analysis on Morrey spaces and applications to Navier-Stokes and other evolution equations. Comm. Partial Differential Equations, 17(9-10):1407-1456, 1992.

[47] M. Taylor. Partial differential equations I, Basic theory, volume 115 of Applied Mathematical Sciences. Springer-Verlag, New York, 1995.

[48] M. Taylor. Partial differential equations. III, volume 117 of Applied Mathematical Sciences. Springer-Verlag, New York, 1997. Nonlinear equations, Corrected reprint of the 1996 original.

[49] M. Taylor. Partial differential equations III, Nonlinear equations, volume 117 of Applied Mathematical Sciences. Springer-Verlag, New York, 1997.

[50] H. Triebel. Characterizations of function spaces on a complete Riemannian manifold with bounded geometry. Math. Nachr., 130:321-346, 1987.

[51] A. Vasy. Propagation of singularities in many-body scattering. Ann. Sci. École Norm. Sup. (4), 34(3):313-402, 2001.

[52] A. Vasy and M. Zworski. Semiclassical estimates in asymptotically Euclidean scattering. Comm. Math. Phys., 212(1):205-217, 2000.

[53] E. Witten. Anti de Sitter space and holography. Adv. Theor. Math. Phys., 2(2):253-291, 1998.

[54] S. Yau. On the heat kernel of a complete Riemannian manifold. J. Math. Pures Appl. (9), 57(2):191-201, 1978.

Pennsylvania State University, Math. Dept., University Park, PA 16802

E-mail address: mazzucat@math.psu.edu

Pennsylvania State University, Math. Dept., University Park, PA 16802

E-mail address: nistor@math.psu.edu 